\title{
Geochemistry of tourmalines associated with iron formation and quartz veins of the Morro da Pedra Preta Formation, Serra do Itaberaba Group (São Paulo, Brazil)
}

\author{
GIANNA M. GARDA ${ }^{1}$, PAUlo BELJAVSKIS ${ }^{2}$, CAETANO JULIANI ${ }^{3}$ and DAILTO SILVA ${ }^{4}$ \\ ${ }^{1}$ Departamento de Geologia Sedimentar e Ambiental, IGc-USP, 05508-080 São Paulo, SP, Brasil \\ ${ }^{2}$ Programa de Recursos Minerais da Pós-Graduação, IGc-USP, 05508-080 São Paulo, SP, Brasil \\ ${ }^{3}$ Departamento de Mineralogia e Geotectônica, IGc-USP, 05508-080 São Paulo, SP, Brasil \\ ${ }^{4}$ Departamento de Metalogênese e Geoquímica, IGe-UNICAMP \\ Cx. Postal 6152, 13083-970 Campinas, SP, Brasil
}

Manuscript received on January 23, 2002; accepted for publication on March 7, 2003; presented by José Moacyr V. COUTINHO

\begin{abstract}
Tourmalines of intermediate schorl-dravite composition occur in iron formation (including metachert and tourmalinites), metasediments, calc-silicate and metabasic/intermediate rocks of the Morro da Pedra Preta Formation, a volcanic-sedimentary sequence of the Serra do Itaberaba Group (northeast of São Paulo City, southeastern Brazil).

The Morro da Pedra Preta Formation is crosscut by quartz veins that contain both intermediate schorl-dravite and an alkali-deficient, $\mathrm{Cr}$-(V-)bearing tourmaline, in which the occupancy of the $\mathrm{X}$-site is $\square_{0.51} \mathrm{Ca}_{0.33} \mathrm{Na}_{0.15}$, characterizing it as intermediate to foitite and magnesiofoitite endmembers. Mg\# values for this tourmaline are higher than those for intermediate schorl-dravite. Raman spectroscopy also confirms the presence of two groups of tourmalines. Stable isotope data indicate sediment waters as fluid sources, rather than fluids from magmatic/post-magmatic sources. Delta ${ }^{18} \mathrm{O}$ compositions for tourmalines, host metachert, and quartz veins are similar, showing that fluid equilibration occurred during crystallization of both quartz and tourmaline. Syngenetic, intermediate schorl-dravite tourmalines were formed under submarine, sedimentaryexhalative conditions; amphibolite-grade metamorphism did not strongly affect their compositions. Younger tourmalines of compositions intermediate to foitite and magnesiofoitite reflect the composition of the host rocks of quartz veins, due to fluid percolation along faults and fractures that caused leaching of $\mathrm{Cr}$ (and V) and the crystallization of these alkali-deficient, $\mathrm{Cr}-(\mathrm{V}-)$ bearing tourmalines.
\end{abstract}

Key words: schorl-dravite series, foitite-magnesiofoitite, Serra do Itaberaba Group, Morro da Pedra Preta Formation.

\section{INTRODUCTION}

Correspondence to: Gianna M. Garda

E-mail: giagarda@usp.br
"The tourmaline minerals in all their aspects" was the topic of the meeting called "Tourmaline 
1997”, held in the Czech Republic in June 1997. Frank C. Hawthorne and Darrell J. Henry presented a proposal for classification of minerals of the tourmaline group, including the alkalideficient tourmaline foitite (McDonald et al. 1993) among the thirteen valid end-members. The classification is based on chemical composition and ordering at the different crystallographic sites of the tourmaline structure (Hawthorne and Henry 1999).

The importance of tourmaline as a petrogenetic indicator has been pointed out by several authors (e.g. Henry and Guidotti 1985, Plimer 1986, 1988, Yavuz 1997) for its ample occurrence as an authigenic mineral in sedimentary and metamorphic rocks. In exploration, dravitic tourmalines have been associated with massive sulfide deposits and exhalites (Plimer 1986, 1987, 1988, Slack 1982, 1996, Slack et al. 1984, Taylor and Slack 1984, Willner 1992); schorlitic compositions have been associated with plutonic rocks (Taylor and Slack 1984), and exhalites (Plimer 1983), and alkali-deficient tourmalines with several environments (Hawthorne et al. 1999).

Tourmaline is a common mineral in the Serra do Itaberaba Group (State of São Paulo, southeastern Brazil), particularly in the basal volcanic-sedimentary sequence named Morro da Pedra Preta Formation (Juliani 1993), which was explored for gold in the 16th and 17th centuries.

Scanning electron microscopy revealed gold disseminated in tourmalinites and associated rocks in the Tapera Grande prospect, and alkali-deficient tourmalines associated with mineralized and barren quartz veins in the Quartzito prospect, both areas found in the Morro da Pedra Preta Formation. Distinct mineralization assemblages charac- terize each area, with gold - pyrite - pyrrhotitechalcopyrite in Tapera Grande (Beljavskis et al. 1999b) and electrum - pyrite - chalcopyritesphalerite-galena-scheelite-molybdenite in Quartzito (Garda et al. 1999).

The association of distinct tourmaline compositions with distinct types of mineralization in the Morro da Pedra Preta Formation led the authors to further investigate the subject, once rock-forming minerals such as amphibole, garnet, staurolite, and sillimanite (among others) proved to be useful for petrogenetic studies of the Serra do Itaberaba Group, but not specifically for mineralization.

\section{The Serra do Itaberaba Group}

Three units compose the Serra do Itaberaba Group (Juliani 1993, Juliani and Beljavskis 1995): the Morro da Pedra Preta, Nhanguçu and Pirucaia Formations.

Lithostratigraphic and chemical characteristics indicate that the deposition of the basal Morro da Pedra Preta Formation began in an ensialic rift that evolved into a deep oceanic basin during the Mesoproterozoic (Juliani et al. 2000). A pile of basic rocks was generated at mid-ocean ridge segments, whereas sediments were deposited in a pelagic environment, with contribution of distal turbidites. Among the rock types, volcanic and volcaniclastic rocks, Algoma-type iron formation, and calc-silicate rocks are recognized. Sulfidation and gold deposition resulted from hydrothermal alteration related to the intrusion of andesitic and dacitic bodies in the volcano-sedimentary sequence under backarc regime (Garda et al. 2002, in press).

The deposition of the Nhanguçu Formation (manganiferous pelites and calc-pelites; carbonatic/calc-silicate rocks, rare (meta) basites 

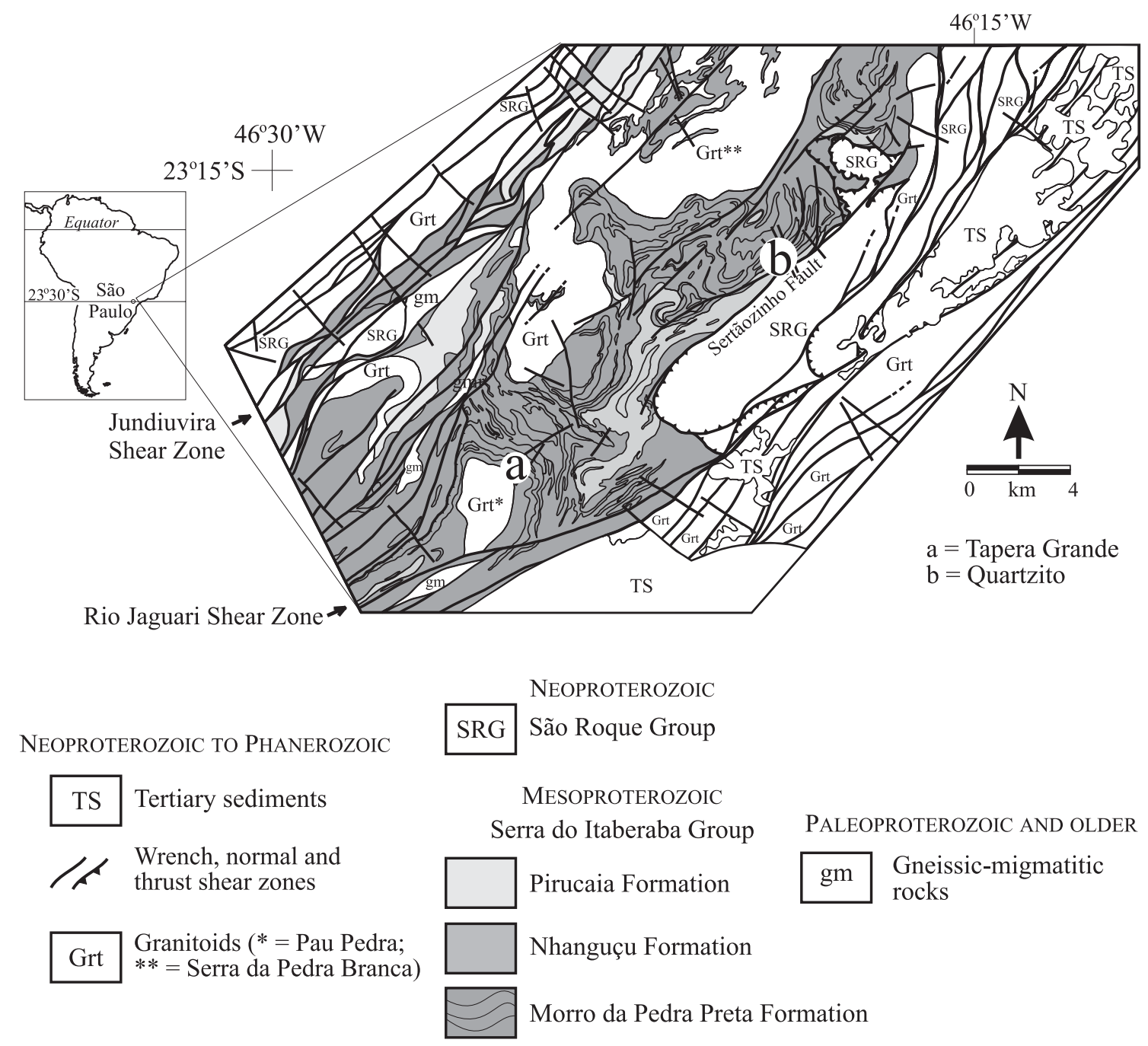

Fig. 1 - Simplified map of Serra do Itaberaba and São Roque Groups (modified from Juliani 1993).

and (meta)volcaniclastic rocks and tourmalinites) followed the closing of the Morro da Pedra Preta basin. On top of the Nhanguçu sequence fine-grained sediments were deposited in progressively shallower waters, and were affected by less intense exhalative volcanic activity.

The Pirucaia Formation (quartzites and quartz schists), possibly coeval with the Nhanguçu Formation, represents continental platform clastic sedimentation that took place in marginal parts of the Serra do Itaberaba basin.
The Serra do Itaberaba Group was metamorphosed to medium amphibolite facies, with the crystallization of kyanite, with later transformation to sillimanite. A second metamorphic event, most likely related to the Brasiliano Cycle, took place in the area, this time reaching the greenschist facies.

Deformed tonalitic and granodioritic granitoids in the region represent in part reworked portions of basement rocks, whereas porphyritic granite and granodiorite bodies, such 
as the Pau Pedra pluton, intruded the Serra do Itaberaba Group in the Neoproterozoic (Brasiliano orogeny). These may be an extension of the nearby syn- to late-orogenic, calc-alkaline, 625 Ma-old Cantareira granitoid occurrence described by Janasi and Ulbrich (1991).

Shear zones such as Rio Jaguari and Jundiuvira and associated faults (e.g. Sertãozinho fault) were developed during transcurrent events that also affected the granitoid rocks.

\section{Tourmalines of the Morro da Pedra Preta FORMATION}

A series of 25 drill cores were obtained from two areas informally named Tapera Grande (NE of the Pau Pedra granitoid body) and Quartzito (W of the Sertãozinho Fault), which sampled large part of the volcano-sedimentary sequence of the Morro da Pedra Preta Formation.

In both study areas (Fig. 1), tourmalines are found in the iron formation (including metachert and tourmalinites), psammites, calcsilicate rocks, basic metatuffs, metavolcaniclastic rocks, amphibolites, and quartz veins.

The iron formation is composed of quartz (50 to $75 \%$ ), magnetite, hematite, iron oxideshydroxides, and variable amounts of tourmaline. In this work, metachert and tourmalinites are considered sub-facies of the iron formation, according to the relative amounts of quartz (which may reach $100 \%$ in metachert) and tourmaline. In the metachert, very darkcolored, zoned, strongly pleochroic (from dark bluish green to light brown) tourmalines occur in an outcrop (sample LJ10A, Fig. 2A) close to SRT-1 drill hole. They form clusters or are aligned according to the general banding of the rock. Subordinately, chlorite also occurs in the metachert, as well as limonite and iron hydroxides.
Tourmaline-rich layers gradually turn into tourmalinites, as described by Slack (1982) and Plimer (1987, 1988). They appear as discontinuous, centimeter- to meter-thick lenses composed of alternating tourmaline-rich and quartzrich bands. Such rocks are fine-grained and contain variable amounts of garnet, hornblende and sericite (after plagioclase). Features like rip-up clasts such as those described by Slack et al. (1984) in the Black Prince mine in Broken Hill (Australia), and Bone (1988) in the Rum Jungle area (Australia) also appear in the Morro da Pedra Preta Formation. The syn-sedimentary nature of the tourmalinites is attested by the $\mathrm{S}_{1}$ foliation, which is marked by the tourmalines and is parallel to bedding. Tourmalinite lenses are sometimes folded, and tourmaline partially recrystallizes in $\mathrm{S}_{2}$, indicating a pre-metamorphic lamination affected by subsequent deformation (Juliani 1993).

Bluish green, strongly pleochroic tourmalines are found in $15 \mathrm{~m}$ thick, folded sequences of biotite to phlogopite quartz schists containing variable amounts of staurolite and garnet. Psammites grade into tourmaline-bearing calcsilicate rocks.

In the basic metatuffs (quartz hornblende schists) brownish green tourmalines appear together with garnet, biotite, chlorite, zoisite, apatite, and carbonate, either as lenses or disseminated in the matrix. They have a more welldeveloped schistosity that can be marked by opaque minerals. A tourmaline-bearing epidotite, considered as resulting from the hydrothermal alteration (carbonatization) of basic metatuffs, occurs at depths of $84 \mathrm{~m}$ in the SRT-1 borehole.

The metavolcaniclastic rocks, which are very similar in composition to the basic metatuffs, contain titanomagnetites altering into ti- 
tanite, abundant plagioclase, quartz, hornblende altering into biotite and chlorite, zoisite, and minor carbonate, apatite, and zircon. Garnet appears in variable amounts, mingled with hornblende. Tourmalines can be as abundant as hornblende. Tourmaline grains present dark, bluish cores and brown rims, and are slightly deformed.

The amphibolites are coarser-grained rocks formed by a felty mass of hornblende, containing biotite, interstitial quartz, and tourmaline that intergrows with amphibole, has dark, bluish cores and brown rims; occasionally it is associated with opaque minerals.

In general associated with shearing, quartz veins crosscut the whole Morro da Pedra Preta sequence. Tourmalines tend to occur concentrated in the contact between veins and country rock (Fig. 2B), associated with sulfides (pyrite and chalcopyrite) of sulfidation stages II, III and IV described by Beljavskis et al. (1999a).

In all rock types, tourmaline is usually finegrained and presents basal sections from 1 to $5 \mathrm{~mm}$ in diameter, except when found arranged in comb texture in tourmaline-rich levels in the metachert, and in this case crystals are larger than $5 \mathrm{~mm}$.

\section{MATERIALS AND METHODS}

The samples chosen for analyses (scanning electron microscopy, Raman spectrometry, X-ray diffraction fluorescence, and oxygen and hydrogen isotopic analysis) were collected from tourmalinite (outcrop T12 - Tapera Grande), tourmaline-rich metachert (outcrops LF10, LJ09, and LJ10 - Tapera Grande; drill hole F01-1B at 3.80m depth - Quartzito), and quartz veins (drill holes SRT3-99 at $54.10 \mathrm{~m}$ depth - Tapera Grande; FQ-112-3a and FQ$112-3 b$ at $18.40 \mathrm{~m}$ depth, and FQ-112-46-3 at
46.23 m depth - Quartzito).

For scanning electron microscopy and Raman spectrometry, polished thin sections of the rock samples were prepared at the Petrology and Rock Technology Laboratory of the Technological Research Institute of São Paulo State (IPT).

Energy dispersive X-ray microanalyses (EDS-SEM) of carbon-coated polished thin sections were carried out at the Scanning Electron Microscope Laboratory of the Geosciences Institute of the Campinas State University (IGeUNICAMP), equipped with a LEO scanning electron microscope model 430 I. The conditions adopted during analyses were: interaction time $=100$ seconds; accelerating voltage $=20 \mathrm{kV}$.

Raman analyses were also carried out at IGe-UNICAMP. The equipment is a laserRaman multi-channel microprobe (CCD T64000 JOBIN-YVON) coupled with a highresolution optical microscope (OLYMPUSBHS) that focuses the laser radiation (Ar laser, line $514.5 \mathrm{~nm}, 80 \mathrm{~mW})$ on the sample $(1 \mu \mathrm{m}$ diameter aperture). A Sony video system helps monitor the laser operation. Beam-sample interaction time adopted was $300 \mathrm{~s}$. The Raman frequencies were calibrated to $1 \mathrm{~cm}^{-1}$ using standard Ne emission lines.

For X-ray diffraction fluorescence, and oxygen and hydrogen stable isotope studies, quartz and tourmaline separates were obtained at the Sample Preparation Laboratory of the Geosciences Institute of São Paulo University - IGc-USP. Crushing of rock chips and screening were followed by heavy liquid separation of quartz and tourmaline, Franz electromagnetic separation of impurities, and hand picking under a binocular microscope.

$\mathrm{X}$-ray diffraction fluorescence analyses 


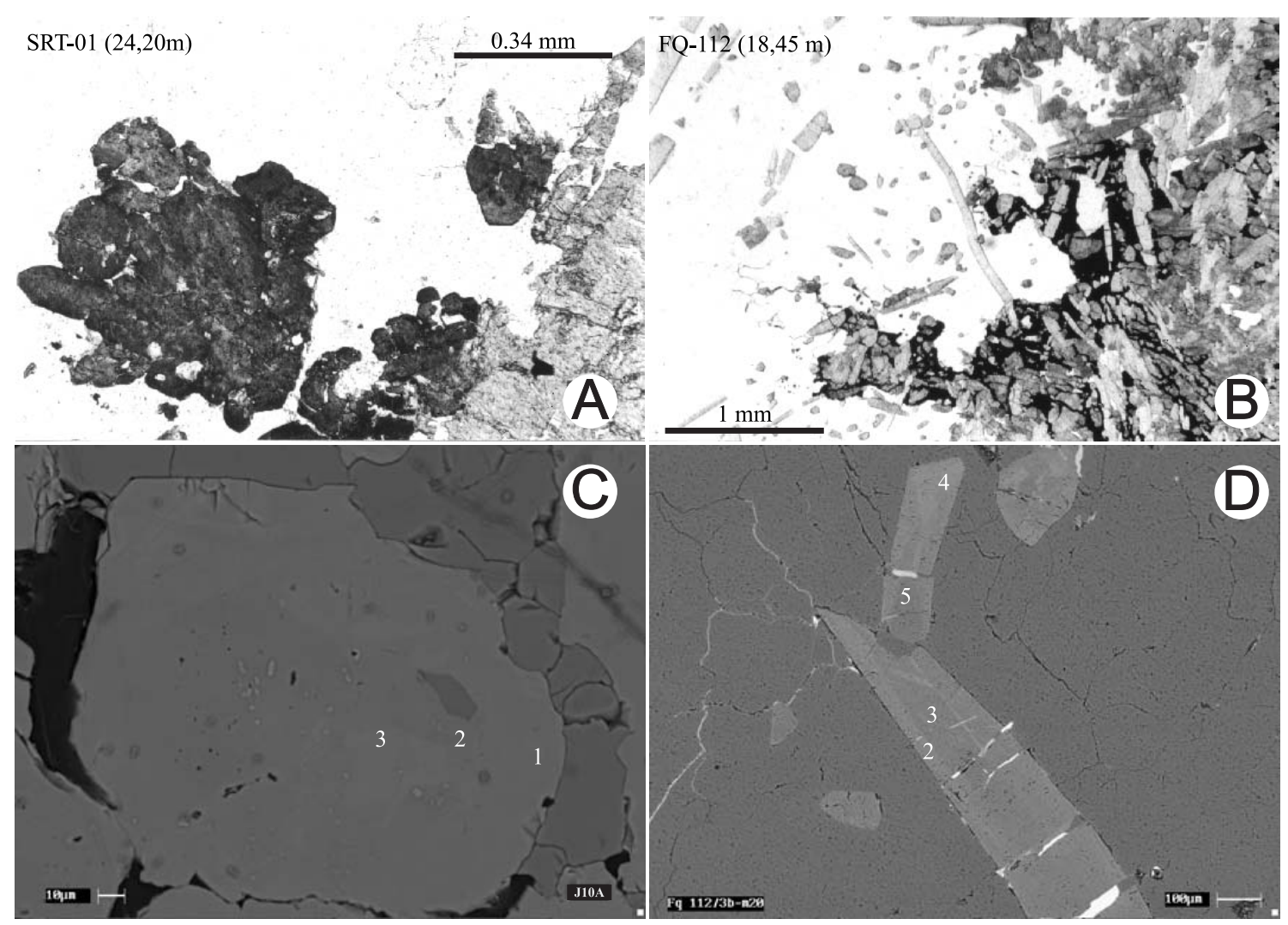

Fig. 2-B\&W photomicrographs of A: very dark, zoned, strongly pleochroic tourmalines from Tapera Grande metachert (plane-polarized light), and B: zoned tourmaline needles of Quartzito quartz vein (plane-polarized light). SEM images of C: zoned tourmaline crystal from Tapera Grande metachert (to points 1, 2 and 3 correspond analyses 19, 20 and 21 in Table I), and D: zoned tourmaline needles of Quartzito quartz vein, also shown in B (to point 2 correspond analyses 63 and 64; to point 3, analyses 67,68 and 71; to point 4, analyses 72, 75 and 76, and point 5, analyses 79 and 80 in Table I).

took place at the XRF Laboratory of IGc-USP. Oxygen and hydrogen stable isotope analyses were carried out at the Scottish Universities Environmental Research Center (East Kilbride, UK), the former with the laser fluorination method (Fallick et al. 1992) and the latter by the conventional method of gas extraction.

\section{RESULTS}

\section{EDS-SEM Mineral ANAlyses}

Table I presents tourmaline compositions obtained by EDS-SEM, with total weight \% of oxides normalized to $100 \%$. Analyses 1 to 21
(Tapera Grande), and 40 and 41 (Quartzito) correspond to tourmalines from metachert; analyses 22 to 30 correspond to tourmalines from a tourmalinite from Tapera Grande (T12); analyses 36 to 39 to tourmalines from a quartz vein from Tapera Grande (SRT3-99), and 44 to 85 to tourmalines from quartz veins of Quartzito (112/3a, 112/3b and 112/46-3).

The structural formulae were calculated according to Henry and Guidotti (1985), assuming 3 boron and 31 oxygen atoms per formula unit (apfu).

Calculations were made using Microsoft 
TABLE I

EDS-SEM analyses of tourmalines from Tapera Grande and Quartzito. Structural formulae calculated on 31 oxygen basis.

\begin{tabular}{|c|c|c|c|c|c|c|c|c|}
\hline \multicolumn{9}{|c|}{$\mathrm{T}$ a $\mathrm{p}$ e $\mathrm{ra}$} \\
\hline Sample & LF10 & LF10 & LF10 & LF10 & LF-10b & LF-10b & LF10 & LJ09 \\
\hline & 1 & 2 & 3 & 4 & 5 & 6 & 11 & 15 \\
\hline $\mathrm{SiO}_{2}$ & 42.97 & 42.90 & 41.10 & 41.61 & 40.41 & 39.55 & 37.58 & 38.48 \\
\hline $\mathrm{TiO}_{2}$ & 0.58 & 0.62 & 0.00 & 0.00 & 0.22 & 0.68 & 0.19 & 0.68 \\
\hline $\mathrm{Al}_{2} \mathrm{O}_{3}$ & 34.01 & 35.10 & 36.71 & 36.59 & 37.62 & 37.87 & 36.94 & 35.81 \\
\hline $\mathrm{FeO}$ & 13.26 & 13.56 & 12.15 & 12.14 & 12.92 & 11.47 & 16.07 & 14.44 \\
\hline $\mathrm{MgO}$ & 5.74 & 4.80 & 5.39 & 5.26 & 5.45 & 6.09 & 4.73 & 6.17 \\
\hline $\mathrm{CaO}$ & 1.11 & 0.60 & 0.45 & 0.59 & 0.49 & 0.92 & 0.47 & 1.01 \\
\hline $\mathrm{Na}_{2} \mathrm{O}$ & 2.34 & 2.42 & 4.19 & 3.81 & 2.89 & 3.43 & 4.02 & 3.41 \\
\hline B & 3.000 & 3.000 & 3.000 & 3.000 & 3.000 & 3.000 & 3.000 & 3.000 \\
\hline $\mathrm{Al}_{\text {total }}$ & 5.777 & 5.954 & 6.243 & 6.209 & 6.394 & 6.432 & 6.440 & 6.195 \\
\hline $\mathrm{Si}_{\text {total }}$ & 6.191 & 6.172 & 5.927 & 5.989 & 5.826 & 5.698 & 5.558 & 5.646 \\
\hline $\mathrm{Si}_{\mathrm{T}}$ & 6.000 & 6.000 & 5.927 & 5.989 & 5.826 & 5.698 & 5.558 & 5.646 \\
\hline $\mathrm{Al}_{\mathrm{T}}$ & 0.000 & 0.000 & 0.073 & 0.011 & 0.174 & 0.302 & 0.442 & 0.354 \\
\hline $\mathrm{Al}_{\mathrm{Z}}$ & 5.777 & 5.954 & 6.000 & 6.000 & 6.000 & 6.000 & 5.998 & 5.841 \\
\hline $\mathrm{Fe}^{3+}$ & 0.223 & 0.046 & 0.000 & 0.000 & 0.000 & 0.000 & 0.002 & 0.159 \\
\hline $\mathrm{Al}_{\mathrm{Y}}$ & 0.000 & 0.000 & 0.170 & 0.197 & 0.219 & 0.130 & 0.000 & 0.000 \\
\hline $\mathrm{SiY}$ & 0.191 & 0.172 & 0.000 & 0.000 & 0.000 & 0.000 & 0.000 & 0.000 \\
\hline $\mathrm{Ti}$ & 0.063 & 0.067 & 0.000 & 0.000 & 0.024 & 0.073 & 0.021 & 0.075 \\
\hline $\mathrm{Fe}_{\text {total }}$ & 1.598 & 1.631 & 1.465 & 1.461 & 1.558 & 1.382 & 1.988 & 1.772 \\
\hline $\mathrm{Fe}^{2+}$ & 1.376 & 1.586 & 1.465 & 1.461 & 1.558 & 1.382 & 1.986 & 1.614 \\
\hline $\mathrm{Mg}$ & 1.231 & 1.029 & 1.160 & 1.127 & 1.172 & 1.308 & 1.044 & 1.349 \\
\hline$Y_{\text {total }}$ & 2.861 & 2.854 & 2.795 & 2.786 & 2.973 & 2.893 & 3.050 & 3.037 \\
\hline $\mathrm{Ca}$ & 0.171 & 0.092 & 0.070 & 0.091 & 0.076 & 0.142 & 0.075 & 0.159 \\
\hline $\mathrm{Na}$ & 0.653 & 0.676 & 1.172 & 1.062 & 0.807 & 0.957 & 1.152 & 0.971 \\
\hline $\mathrm{X}_{\text {total }}$ & 0.824 & 0.768 & 1.242 & 1.152 & 0.883 & 1.099 & 1.228 & 1.130 \\
\hline [] & 0.176 & 0.232 & -0.242 & -0.152 & 0.117 & -0.099 & -0.228 & -0.130 \\
\hline Total & 15.684 & 15.622 & 16.037 & 15.938 & 15.857 & 15.991 & 16.278 & 16.167 \\
\hline $\mathrm{R} 1$ & 0.824 & 0.768 & 1.242 & 1.152 & 0.883 & 1.099 & 1.228 & 1.130 \\
\hline $\mathrm{R} 2$ & 2.607 & 2.615 & 2.625 & 2.589 & 2.730 & 2.690 & 3.029 & 2.962 \\
\hline $\mathrm{R} 1+\mathrm{R} 2$ & 3.431 & 3.383 & 3.867 & 3.741 & 3.613 & 3.788 & 4.257 & 4.093 \\
\hline R3 & 5.861 & 6.044 & 6.243 & 6.209 & 6.426 & 6.529 & 6.468 & 6.295 \\
\hline Mg\# & 0.435 & 0.387 & 0.442 & 0.436 & 0.429 & 0.486 & 0.344 & 0.432 \\
\hline
\end{tabular}


TABLE I (continuation)

\begin{tabular}{|c|c|c|c|c|c|c|c|c|}
\hline \multicolumn{9}{|c|}{$\mathrm{T}$ a $\mathrm{p} \mathrm{e} \mathrm{ra}$} \\
\hline Sample & LJ09 & LJ09 & LJ09 & LJ10A & LJ10A & LJ10A & $\mathrm{T} 12$ & $\mathrm{~T} 12$ \\
\hline & 16 & 17 & 18 & 19 & 20 & 21 & 22 & 23 \\
\hline $\mathrm{SiO}_{2}$ & 39.72 & 41.58 & 43.31 & 39.50 & 39.89 & 39.19 & 40.28 & 45.07 \\
\hline $\mathrm{TiO}_{2}$ & 0.83 & 1.12 & 0.25 & 1.00 & 0.32 & 0.51 & 0.93 & 0.19 \\
\hline $\mathrm{Al}_{2} \mathrm{O}_{3}$ & 36.60 & 32.09 & 35.46 & 36.13 & 37.26 & 34.51 & 38.21 & 36.58 \\
\hline $\mathrm{FeO}$ & 13.23 & 17.49 & 13.04 & 14.41 & 12.84 & 17.07 & 15.19 & 8.13 \\
\hline $\mathrm{MgO}$ & 5.93 & 4.46 & 5.15 & 5.29 & 6.16 & 4.81 & 2.81 & 7.36 \\
\hline $\mathrm{CaO}$ & 0.89 & 1.41 & 0.52 & 0.96 & 0.85 & 1.02 & 0.78 & 0.41 \\
\hline $\mathrm{Na}_{2} \mathrm{O}$ & 2.80 & 1.85 & 2.27 & 2.71 & 2.67 & 2.89 & 1.81 & 2.26 \\
\hline B & 3.000 & 3.000 & 3.000 & 3.000 & 3.000 & 3.000 & 3.000 & 3.000 \\
\hline $\mathrm{Al}_{\text {total }}$ & 6.256 & 5.578 & 5.989 & 6.212 & 6.347 & 6.022 & 6.530 & 6.020 \\
\hline $\mathrm{Si}_{\text {total }}$ & 5.759 & 6.130 & 6.204 & 5.761 & 5.763 & 5.801 & 5.839 & 6.291 \\
\hline $\mathrm{Si}_{\mathrm{T}}$ & 5.759 & 6.000 & 6.000 & 5.761 & 5.763 & 5.801 & 5.839 & 6.000 \\
\hline $\mathrm{Al}_{\mathrm{T}}$ & 0.241 & 0.000 & 0.000 & 0.239 & 0.237 & 0.199 & 0.161 & 0.000 \\
\hline $\mathrm{Al}_{\mathrm{Z}}$ & 6.000 & 5.578 & 5.989 & 5.973 & 6.000 & 5.823 & 6.000 & 6.000 \\
\hline $\mathrm{Fe}^{3+}$ & 0.000 & 0.422 & 0.011 & 0.027 & 0.000 & 0.177 & 0.000 & 0.000 \\
\hline $\mathrm{Al}_{\mathrm{Y}}$ & 0.015 & 0.000 & 0.000 & 0.000 & 0.110 & 0.000 & 0.369 & 0.020 \\
\hline SiY & 0.000 & 0.130 & 0.204 & 0.000 & 0.000 & 0.000 & 0.000 & 0.291 \\
\hline $\mathrm{Ti}$ & 0.090 & 0.125 & 0.027 & 0.110 & 0.035 & 0.057 & 0.102 & 0.020 \\
\hline $\mathrm{Fe}_{\text {total }}$ & 1.604 & 2.157 & 1.563 & 1.757 & 1.551 & 2.114 & 1.842 & 0.949 \\
\hline $\mathrm{Fe}^{2+}$ & 1.604 & 1.735 & 1.551 & 1.731 & 1.551 & 1.937 & 1.842 & 0.949 \\
\hline $\mathrm{Mg}$ & 1.281 & 0.979 & 1.099 & 1.149 & 1.326 & 1.061 & 0.606 & 1.531 \\
\hline$Y_{\text {total }}$ & 2.991 & 2.969 & 2.881 & 2.990 & 3.022 & 3.054 & 2.919 & 2.812 \\
\hline $\mathrm{Ca}$ & 0.138 & 0.222 & 0.080 & 0.150 & 0.132 & 0.162 & 0.121 & 0.061 \\
\hline $\mathrm{Na}$ & 0.787 & 0.529 & 0.630 & 0.766 & 0.749 & 0.829 & 0.509 & 0.613 \\
\hline$X_{\text {total }}$ & 0.925 & 0.751 & 0.710 & 0.916 & 0.881 & 0.991 & 0.629 & 0.673 \\
\hline [] & 0.075 & 0.249 & 0.290 & 0.084 & 0.119 & 0.009 & 0.371 & 0.327 \\
\hline Total & 15.916 & 15.721 & 15.590 & 15.906 & 15.903 & 16.045 & 15.549 & 15.485 \\
\hline R1 & 0.925 & 0.751 & 0.710 & 0.916 & 0.881 & 0.991 & 0.629 & 0.673 \\
\hline R2 & 2.886 & 2.714 & 2.650 & 2.880 & 2.877 & 2.997 & 2.448 & 2.480 \\
\hline $\mathrm{R} 1+\mathrm{R} 2$ & 3.811 & 3.466 & 3.360 & 3.796 & 3.758 & 3.988 & 3.078 & 3.154 \\
\hline R3 & 6.376 & 5.744 & 6.024 & 6.359 & 6.393 & 6.098 & 6.666 & 6.047 \\
\hline Mg\# & 0.444 & 0.312 & 0.413 & 0.395 & 0.461 & 0.334 & 0.248 & 0.617 \\
\hline
\end{tabular}


TABLE I (continuation)

\begin{tabular}{|c|c|c|c|c|c|c|c|c|}
\hline \multicolumn{9}{|c|}{$\mathrm{T}$ a $\mathrm{p}$ e $\mathrm{ra}$} \\
\hline Sample & $\mathrm{T} 12$ & $\mathrm{~T} 12$ & $\mathrm{~T} 12 \mathrm{a}$ & $\mathrm{T} 12 \mathrm{a}$ & T12a & T12a & SRT3-99 & SRT3-99 \\
\hline & 24 & 25 & 27 & 28 & 29 & 30 & 36 & 37 \\
\hline $\mathrm{SiO}_{2}$ & 44.48 & 44.38 & 42.07 & 40.74 & 40.73 & 43.26 & 41.35 & 42.48 \\
\hline $\mathrm{TiO}_{2}$ & 0.82 & 0.37 & 0.68 & 1.02 & 1.13 & 0.73 & 1.05 & 0.86 \\
\hline $\mathrm{Al}_{2} \mathrm{O}_{3}$ & 35.44 & 36.47 & 36.39 & 36.06 & 36.17 & 36.81 & 31.54 & 34.89 \\
\hline $\mathrm{FeO}$ & 10.28 & 10.70 & 13.27 & 15.11 & 15.89 & 10.48 & 18.11 & 11.93 \\
\hline $\mathrm{MgO}$ & 6.36 & 5.69 & 5.68 & 4.29 & 3.46 & 5.49 & 4.64 & 5.89 \\
\hline $\mathrm{CaO}$ & 0.47 & 0.39 & 0.00 & 0.66 & 0.50 & 0.38 & 1.27 & 1.08 \\
\hline $\mathrm{Na}_{2} \mathrm{O}$ & 2.16 & 1.99 & 1.91 & 2.13 & 2.12 & 2.86 & 2.04 & 2.86 \\
\hline $\mathrm{B}$ & 3.000 & 3.000 & 3.000 & 3.000 & 3.000 & 3.000 & 3.000 & 3.000 \\
\hline $\mathrm{Al}_{\text {total }}$ & 5.899 & 6.068 & 6.150 & 6.178 & 6.212 & 6.151 & 5.507 & 5.906 \\
\hline $\mathrm{Si}_{\text {total }}$ & 6.280 & 6.264 & 6.029 & 5.921 & 5.935 & 6.133 & 6.123 & 6.099 \\
\hline $\mathrm{Si}_{\mathrm{T}}$ & 6.000 & 6.000 & 6.000 & 5.921 & 5.935 & 6.000 & 6.000 & 6.000 \\
\hline $\mathrm{Al}_{\mathrm{T}}$ & 0.000 & 0.000 & 0.000 & 0.079 & 0.065 & 0.000 & 0.000 & 0.000 \\
\hline $\mathrm{Al}_{\mathrm{Z}}$ & 5.899 & 6.000 & 6.000 & 6.000 & 6.000 & 6.000 & 5.507 & 5.906 \\
\hline $\mathrm{Fe}^{3+}$ & 0.101 & 0.000 & 0.000 & 0.000 & 0.000 & 0.000 & 0.493 & 0.094 \\
\hline $\mathrm{AlY}_{\mathrm{Y}}$ & 0.000 & 0.068 & 0.150 & 0.100 & 0.147 & 0.151 & 0.000 & 0.000 \\
\hline SiY & 0.280 & 0.264 & 0.029 & 0.000 & 0.000 & 0.133 & 0.123 & 0.099 \\
\hline $\mathrm{Ti}$ & 0.087 & 0.040 & 0.074 & 0.111 & 0.123 & 0.077 & 0.117 & 0.093 \\
\hline $\mathrm{Fe}_{\text {total }}$ & 1.214 & 1.263 & 1.590 & 1.837 & 1.936 & 1.242 & 2.242 & 1.432 \\
\hline $\mathrm{Fe}^{2+}$ & 1.113 & 1.263 & 1.590 & 1.837 & 1.936 & 1.242 & 1.749 & 1.338 \\
\hline $\mathrm{Mg}$ & 1.337 & 1.197 & 1.214 & 0.928 & 0.752 & 1.160 & 1.024 & 1.260 \\
\hline$Y_{\text {total }}$ & 2.818 & 2.831 & 3.056 & 2.976 & 2.958 & 2.764 & 3.013 & 2.790 \\
\hline $\mathrm{Ca}$ & 0.070 & 0.059 & 0.000 & 0.103 & 0.078 & 0.057 & 0.201 & 0.167 \\
\hline $\mathrm{Na}$ & 0.590 & 0.544 & 0.531 & 0.600 & 0.600 & 0.786 & 0.585 & 0.797 \\
\hline $\mathrm{X}_{\text {total }}$ & 0.661 & 0.604 & 0.531 & 0.703 & 0.678 & 0.843 & 0.786 & 0.964 \\
\hline [] & 0.339 & 0.396 & 0.469 & 0.297 & 0.322 & 0.157 & 0.214 & 0.036 \\
\hline Total & 15.478 & 15.435 & 15.588 & 15.679 & 15.636 & 15.607 & 15.799 & 15.754 \\
\hline $\mathrm{R} 1$ & 0.661 & 0.604 & 0.531 & 0.703 & 0.678 & 0.843 & 0.786 & 0.964 \\
\hline R2 & 2.451 & 2.460 & 2.804 & 2.765 & 2.688 & 2.402 & 2.773 & 2.598 \\
\hline $\mathrm{R} 1+\mathrm{R} 2$ & 3.111 & 3.064 & 3.335 & 3.468 & 3.366 & 3.245 & 3.559 & 3.562 \\
\hline $\mathrm{R} 3$ & 6.015 & 6.120 & 6.248 & 6.326 & 6.376 & 6.254 & 5.662 & 6.029 \\
\hline $\mathrm{Mg \#}$ & 0.524 & 0.487 & 0.433 & 0.336 & 0.280 & 0.483 & 0.313 & 0.468 \\
\hline
\end{tabular}


TABLE I (continuation)

\begin{tabular}{|c|c|c|c|c|c|c|}
\hline \multicolumn{3}{|c|}{$\mathrm{T}$ a $\mathrm{p}$ e $\mathrm{ra} \quad \mathrm{Gr}$ a $\mathrm{n} \mathrm{de}$} & \multicolumn{4}{|c|}{$\mathrm{Quartz} \mathrm{i}$ to } \\
\hline Sample & SRT-3-99 & SRT-3-99 & F01-1B & F01-1B & $112 / 3 a-m 10$ & 112/3a-m10 \\
\hline & 38 & 39 & 40 & 41 & 44 & 47 \\
\hline $\mathrm{SiO}_{2}$ & 38.38 & 39.40 & 40.05 & 39.63 & 41.29 & 41.36 \\
\hline $\mathrm{TiO}_{2}$ & 1.11 & 0.72 & 0.76 & 0.00 & 0.69 & 0.69 \\
\hline $\mathrm{Al}_{2} \mathrm{O}_{3}$ & 34.84 & 35.45 & 37.52 & 39.48 & 37.48 & 37.51 \\
\hline $\mathrm{FeO}$ & 16.98 & 15.37 & 11.86 & 13.25 & 9.08 & 8.94 \\
\hline $\mathrm{MgO}$ & 5.34 & 5.32 & 5.55 & 3.75 & 9.66 & 9.60 \\
\hline $\mathrm{CaO}$ & 1.17 & 1.08 & 0.90 & 0.51 & 1.80 & 1.90 \\
\hline $\mathrm{Na}_{2} \mathrm{O}$ & 2.17 & 2.67 & 3.37 & 3.38 & $<$ d.l. & $<$ d.l. \\
\hline $\mathrm{Cr}_{2} \mathrm{O}_{3}$ & n.d. & n.d. & n.d. & n.d. & $<$ d.l. & < d.1. \\
\hline B & 3.000 & 3.000 & 3.000 & 3.000 & 3.000 & 3.000 \\
\hline $\mathrm{Al}_{\text {total }}$ & 6.078 & 6.128 & 6.375 & 6.724 & 6.228 & 6.230 \\
\hline $\mathrm{Si}_{\text {total }}$ & 5.679 & 5.777 & 5.772 & 5.726 & 5.820 & 5.827 \\
\hline $\mathrm{Si}_{\mathrm{T}}$ & 5.679 & 5.777 & 5.772 & 5.726 & 5.820 & 5.827 \\
\hline $\mathrm{Al}_{\mathrm{T}}$ & 0.321 & 0.223 & 0.228 & 0.274 & 0.180 & 0.173 \\
\hline $\mathrm{Al}_{\mathrm{Z}}$ & 5.757 & 5.905 & 6.000 & 6.000 & 6.000 & 6.000 \\
\hline $\mathrm{Fe}^{3+}$ & 0.243 & 0.095 & 0.000 & 0.000 & 0.000 & 0.000 \\
\hline $\mathrm{Al}_{\mathrm{Y}}$ & 0.000 & 0.000 & 0.147 & 0.450 & 0.048 & 0.056 \\
\hline $\mathrm{Si}_{Y}$ & 0.000 & 0.000 & 0.000 & 0.000 & 0.000 & 0.000 \\
\hline $\mathrm{Ti}$ & 0.124 & 0.079 & 0.082 & 0.000 & 0.073 & 0.073 \\
\hline $\mathrm{Fe}_{\text {total }}$ & 2.101 & 1.884 & 1.429 & 1.601 & 1.070 & 1.053 \\
\hline $\mathrm{Fe}^{2+}$ & 1.858 & 1.789 & 1.429 & 1.601 & 1.070 & 1.053 \\
\hline $\mathrm{Mg}$ & 1.178 & 1.162 & 1.192 & 0.808 & 2.030 & 2.016 \\
\hline $\mathrm{Cr}$ & - & - & - & - & - & - \\
\hline$Y_{\text {total }}$ & 3.160 & 3.030 & 2.850 & 2.859 & 3.221 & 3.198 \\
\hline $\mathrm{Ca}$ & 0.186 & 0.170 & 0.139 & 0.079 & 0.272 & 0.288 \\
\hline $\mathrm{Na}$ & 0.624 & 0.760 & 0.941 & 0.947 & 0.000 & 0.000 \\
\hline $\mathrm{X}_{\text {total }}$ & 0.810 & 0.930 & 1.079 & 1.026 & 0.272 & 0.288 \\
\hline [] & 0.190 & 0.070 & -0.079 & -0.026 & 0.728 & 0.712 \\
\hline Total & 15.970 & 15.960 & 15.929 & 15.885 & 15.493 & 15.486 \\
\hline R1 & 0.810 & 0.930 & 1.079 & 1.026 & 0.272 & 0.288 \\
\hline R2 & 3.036 & 2.951 & 2.621 & 2.409 & 3.100 & 3.069 \\
\hline $\mathrm{R} 1+\mathrm{R} 2$ & 3.846 & 3.881 & 3.700 & 3.435 & 3.372 & 3.356 \\
\hline R3 & 6.243 & 6.233 & 6.484 & 6.724 & 6.325 & 6.326 \\
\hline $\mathrm{Mg \#}$ & 0.359 & 0.381 & 0.455 & 0.335 & 0.655 & 0.657 \\
\hline
\end{tabular}

n.d. $=$ not determined $;<$ d.1. = below detection limit 
TABLE I (continuation)

\begin{tabular}{|c|c|c|c|c|c|c|}
\hline \multicolumn{7}{|c|}{$\mathrm{Qu} \mathrm{a} \mathrm{rtzirl}^{\mathrm{z}}$} \\
\hline Sample & $\begin{array}{c}\text { 112/3a-m10 } \\
48 \\
\end{array}$ & $\begin{array}{c}112 / 3 \mathrm{a}-\mathrm{m} 10 \\
51\end{array}$ & $\begin{array}{c}112 / 3 \mathrm{a}-\mathrm{m} 10 \\
52\end{array}$ & $\begin{array}{c}112 / 3 \mathrm{a}-\mathrm{m} 10 \\
53\end{array}$ & $\begin{array}{c}\text { 112/3a-m10 } \\
56 \\
\end{array}$ & $\begin{array}{c}112 / 3 \mathrm{~b}-\mathrm{m} 20 \\
63 \\
\end{array}$ \\
\hline $\mathrm{SiO}_{2}$ & 41.25 & 42.33 & 42.21 & 41.50 & 42.02 & 41.34 \\
\hline $\mathrm{TiO}_{2}$ & 0.95 & 0.00 & 0.23 & 0.57 & 0.31 & 0.57 \\
\hline $\mathrm{Al}_{2} \mathrm{O}_{3}$ & 36.26 & 38.88 & 38.82 & 37.90 & 38.88 & 38.52 \\
\hline $\mathrm{FeO}$ & 10.29 & 8.98 & 8.94 & 8.48 & 8.74 & 8.84 \\
\hline $\mathrm{MgO}$ & 9.11 & 8.83 & 8.83 & 9.78 & 9.08 & 8.92 \\
\hline $\mathrm{CaO}$ & 2.13 & 0.97 & 0.97 & 1.78 & 0.97 & 1.45 \\
\hline $\mathrm{Na}_{2} \mathrm{O}$ & $<$ d.l. & $<$ d.l. & $<$ d.l. & $<$ d.l. & $<$ d.l. & $<$ d.l. \\
\hline $\mathrm{Cr}_{2} \mathrm{O}_{3}$ & $<$ d.1. & $<$ d.l. & $<$ d.l. & $<$ d.l. & $<$ d.l. & 0.36 \\
\hline B & 3.000 & 3.000 & 3.000 & 3.000 & 3.000 & 3.000 \\
\hline $\mathrm{Al}_{\text {total }}$ & 6.071 & 6.413 & 6.405 & 6.275 & 6.413 & 6.382 \\
\hline $\mathrm{Si}_{\text {total }}$ & 5.858 & 5.922 & 5.906 & 5.828 & 5.878 & 5.810 \\
\hline $\mathrm{Si}_{\mathrm{T}}$ & 5.858 & 5.922 & 5.906 & 5.828 & 5.878 & 5.810 \\
\hline $\mathrm{Al}_{\mathrm{T}}$ & 0.142 & 0.078 & 0.094 & 0.172 & 0.122 & 0.190 \\
\hline $\mathrm{Al}_{\mathrm{Z}}$ & 5.929 & 6.000 & 6.000 & 6.000 & 6.000 & 6.000 \\
\hline $\mathrm{Fe}^{3+}$ & 0.071 & 0.000 & 0.000 & 0.000 & 0.000 & 0.000 \\
\hline $\mathrm{Al}_{\mathrm{Y}}$ & 0.000 & 0.335 & 0.310 & 0.103 & 0.290 & 0.192 \\
\hline $\mathrm{Si}_{Y}$ & 0.000 & 0.000 & 0.000 & 0.000 & 0.000 & 0.000 \\
\hline $\mathrm{Ti}$ & 0.101 & 0.000 & 0.024 & 0.061 & 0.032 & 0.061 \\
\hline $\mathrm{Fe}_{\text {total }}$ & 1.222 & 1.050 & 1.046 & 0.996 & 1.022 & 1.039 \\
\hline $\mathrm{Fe}^{2+}$ & 1.151 & 1.050 & 1.046 & 0.996 & 1.022 & 1.039 \\
\hline $\mathrm{Mg}$ & 1.928 & 1.841 & 1.841 & 2.047 & 1.894 & 1.867 \\
\hline $\mathrm{Cr}$ & - & - & - & - & - & 0.040 \\
\hline$Y_{\text {total }}$ & 3.180 & 3.226 & 3.222 & 3.207 & 3.238 & 3.200 \\
\hline $\mathrm{Ca}$ & 0.325 & 0.145 & 0.145 & 0.267 & 0.145 & 0.218 \\
\hline $\mathrm{Na}$ & 0.000 & 0.000 & 0.000 & 0.000 & 0.000 & 0.000 \\
\hline$X_{\text {total }}$ & 0.325 & 0.145 & 0.145 & 0.267 & 0.145 & 0.218 \\
\hline [] & 0.675 & 0.855 & 0.855 & 0.733 & 0.855 & 0.782 \\
\hline Total & 15.505 & 15.372 & 15.368 & 15.474 & 15.384 & 15.418 \\
\hline $\mathrm{R} 1$ & 0.325 & 0.145 & 0.145 & 0.267 & 0.145 & 0.218 \\
\hline $\mathrm{R} 2$ & 3.079 & 2.892 & 2.888 & 3.043 & 2.916 & 2.907 \\
\hline $\mathrm{R} 1+\mathrm{R} 2$ & 3.403 & 3.037 & 3.033 & 3.310 & 3.061 & 3.125 \\
\hline R3 & 6.206 & 6.413 & 6.437 & 6.356 & 6.456 & 6.463 \\
\hline Mg\# & 0.612 & 0.637 & 0.638 & 0.673 & 0.650 & 0.642 \\
\hline
\end{tabular}

n.d. $=$ not determined; $<$ d.1. = below detection limit 
TABLE I (continuation)

\begin{tabular}{|c|c|c|c|c|c|c|}
\hline \multicolumn{7}{|c|}{$\mathrm{Quartz} \mathrm{ito}$} \\
\hline Sample & $\begin{array}{c}112 / 3 b-m 20 \\
64\end{array}$ & $\begin{array}{c}112 / 3 b-\mathrm{m} 20 \\
67\end{array}$ & $\begin{array}{c}112 / 3 b-\mathrm{m} 20 \\
68\end{array}$ & $\begin{array}{c}112 / 3 b-\mathrm{m} 20 \\
71\end{array}$ & $\begin{array}{c}112 / 3 b-m 20 \\
72\end{array}$ & $\begin{array}{c}112 / 3 b-m 20 \\
75\end{array}$ \\
\hline $\mathrm{SiO}_{2}$ & 41.35 & 42.07 & 41.93 & 41.73 & 41.84 & 41.82 \\
\hline $\mathrm{TiO}_{2}$ & 0.38 & 0.27 & 0.35 & 0.27 & 0.27 & 0.38 \\
\hline $\mathrm{Al}_{2} \mathrm{O}_{3}$ & 38.92 & 39.37 & 38.39 & 39.18 & 39.55 & 38.89 \\
\hline $\mathrm{FeO}$ & 8.81 & 8.12 & 8.38 & 8.22 & 8.37 & 8.48 \\
\hline $\mathrm{MgO}$ & 8.81 & 8.82 & 9.45 & 9.09 & 8.81 & 8.98 \\
\hline $\mathrm{CaO}$ & 1.40 & 1.11 & 1.32 & 1.19 & 1.16 & 1.18 \\
\hline $\mathrm{Na}_{2} \mathrm{O}$ & $<\mathrm{d} .1$. & $<\mathrm{d} .1$. & $<$ d.l. & $<$ d.l. & $<$ d.l. & $<$ d. 1. \\
\hline $\mathrm{Cr}_{2} \mathrm{O}_{3}$ & 0.33 & 0.26 & 0.18 & 0.33 & 0.00 & 0.26 \\
\hline $\mathrm{V}_{2} \mathrm{O}_{5}$ & $<$ d.l. & $<\mathrm{d} .1$. & $<$ d.l. & $<$ d.l. & $<$ d.l. & $<$ d.l. \\
\hline $\mathrm{B}$ & 3.000 & 3.000 & 3.000 & 3.000 & 3.000 & 3.000 \\
\hline $\mathrm{Al}_{\text {total }}$ & 6.443 & 6.477 & 6.337 & 6.458 & 6.513 & 6.417 \\
\hline $\mathrm{Si}_{\text {total }}$ & 5.806 & 5.870 & 5.870 & 5.835 & 5.845 & 5.854 \\
\hline $\mathrm{Si}_{\mathrm{T}}$ & 5.806 & 5.870 & 5.870 & 5.835 & 5.845 & 5.854 \\
\hline $\mathrm{Al}_{\mathrm{T}}$ & 0.194 & 0.130 & 0.130 & 0.165 & 0.155 & 0.146 \\
\hline $\mathrm{Al}_{\mathrm{Z}}$ & 6.000 & 6.000 & 6.000 & 6.000 & 6.000 & 6.000 \\
\hline $\mathrm{Fe}^{3+}$ & 0.000 & 0.000 & 0.000 & 0.000 & 0.000 & 0.000 \\
\hline $\mathrm{Al}_{\mathrm{Y}}$ & 0.249 & 0.346 & 0.207 & 0.293 & 0.358 & 0.271 \\
\hline $\mathrm{Si}_{Y}$ & 0.000 & 0.000 & 0.000 & 0.000 & 0.000 & 0.000 \\
\hline $\mathrm{Ti}$ & 0.040 & 0.028 & 0.036 & 0.028 & 0.028 & 0.040 \\
\hline $\mathrm{Fe}_{\text {total }}$ & 1.035 & 0.947 & 0.982 & 0.961 & 0.978 & 0.993 \\
\hline $\mathrm{Fe}^{2+}$ & 1.035 & 0.947 & 0.982 & 0.961 & 0.978 & 0.993 \\
\hline $\mathrm{Mg}$ & 1.843 & 1.834 & 1.971 & 1.893 & 1.833 & 1.873 \\
\hline $\mathrm{Cr}$ & 0.036 & 0.028 & 0.020 & 0.036 & 0.000 & 0.028 \\
\hline $\mathrm{V}$ & - & - & - & - & - & - \\
\hline$Y_{\text {total }}$ & 3.204 & 3.184 & 3.217 & 3.212 & 3.197 & 3.205 \\
\hline $\mathrm{Ca}$ & 0.210 & 0.165 & 0.198 & 0.178 & 0.174 & 0.178 \\
\hline $\mathrm{Na}$ & 0.000 & 0.000 & 0.000 & 0.000 & 0.000 & 0.000 \\
\hline $\mathrm{X}_{\text {total }}$ & 0.210 & 0.165 & 0.198 & 0.178 & 0.174 & 0.178 \\
\hline [] & 0.790 & 0.835 & 0.802 & 0.822 & 0.826 & 0.822 \\
\hline Total & 15.414 & 15.349 & 15.415 & 15.390 & 15.370 & 15.383 \\
\hline $\mathrm{R} 1$ & 0.210 & 0.165 & 0.198 & 0.178 & 0.174 & 0.178 \\
\hline $\mathrm{R} 2$ & 2.878 & 2.781 & 2.953 & 2.854 & 2.811 & 2.866 \\
\hline $\mathrm{R} 1+\mathrm{R} 2$ & 3.088 & 2.947 & 3.151 & 3.032 & 2.984 & 3.044 \\
\hline R3 & 6.496 & 6.514 & 6.385 & 6.496 & 6.551 & 6.471 \\
\hline $\mathrm{Mg \#}$ & 0.640 & 0.659 & 0.668 & 0.663 & 0.652 & 0.653 \\
\hline
\end{tabular}

n.d. $=$ not determined $;<$ d.l. = below detection limit 
TABLE I (continuation)

\begin{tabular}{|c|c|c|c|c|c|}
\hline \multicolumn{6}{|c|}{$\mathrm{Quartz} \mathrm{i} \mathrm{t} \mathrm{o}$} \\
\hline Sample & $\begin{array}{c}112 / 3 b-m 20 \\
76\end{array}$ & $\begin{array}{c}112 / 3 b-m 20 \\
79\end{array}$ & $\begin{array}{c}112 / 3 b-m 20 \\
80\end{array}$ & $\begin{array}{c}112 / 46-3 \\
84\end{array}$ & $\begin{array}{c}112 / 46-3 \\
85\end{array}$ \\
\hline $\mathrm{SiO}_{2}$ & 41.89 & 42.60 & 42.43 & 41.78 & 41.27 \\
\hline $\mathrm{TiO}_{2}$ & 0.35 & 0.00 & 0.00 & 0.00 & 0.00 \\
\hline $\mathrm{Al}_{2} \mathrm{O}_{3}$ & 39.21 & 39.02 & 38.87 & 39.18 & 39.53 \\
\hline $\mathrm{FeO}$ & 8.42 & 8.04 & 8.10 & 7.10 & 7.09 \\
\hline $\mathrm{MgO}$ & 8.69 & 9.60 & 9.58 & 10.13 & 10.33 \\
\hline $\mathrm{CaO}$ & 1.27 & 0.73 & 0.76 & 1.81 & 1.77 \\
\hline $\mathrm{Na}_{2} \mathrm{O}$ & $<$ d.l. & $<$ d.l. & $<$ d.l. & $<$ d.l. & $<$ d.l. \\
\hline $\mathrm{Cr}_{2} \mathrm{O}_{3}$ & 0.18 & $<$ d.l. & $<$ d.l. & $<$ d.l. & $<$ d.l. \\
\hline $\mathrm{V}_{2} \mathrm{O}_{5}$ & $<$ d.l. & $<$ d.1. & 0.26 & $<$ d.l. & $<$ d.l. \\
\hline $\mathrm{B}$ & 3.000 & 3.000 & 3.000 & 3.000 & 3.000 \\
\hline $\mathrm{Al}_{\text {total }}$ & 6.465 & 6.403 & 6.383 & 6.437 & 6.499 \\
\hline $\mathrm{Si}_{\text {total }}$ & 5.858 & 5.929 & 5.910 & 5.822 & 5.756 \\
\hline $\mathrm{Si}_{\mathrm{T}}$ & 5.858 & 5.929 & 5.910 & 5.822 & 5.756 \\
\hline $\mathrm{Al}_{\mathrm{T}}$ & 0.142 & 0.071 & 0.090 & 0.178 & 0.244 \\
\hline $\mathrm{Al}_{\mathrm{Z}}$ & 6.000 & 6.000 & 6.000 & 6.000 & 6.000 \\
\hline $\mathrm{Fe}^{3+}$ & 0.000 & 0.000 & 0.000 & 0.000 & 0.000 \\
\hline $\mathrm{Al}_{\mathrm{Y}}$ & 0.322 & 0.332 & 0.293 & 0.259 & 0.255 \\
\hline $\mathrm{Si}_{Y}$ & 0.000 & 0.000 & 0.000 & 0.000 & 0.000 \\
\hline $\mathrm{Ti}$ & 0.036 & 0.000 & 0.000 & 0.000 & 0.000 \\
\hline $\mathrm{Fe}_{\text {total }}$ & 0.984 & 0.936 & 0.944 & 0.828 & 0.827 \\
\hline $\mathrm{Fe}^{2+}$ & 0.984 & 0.936 & 0.944 & 0.828 & 0.827 \\
\hline $\mathrm{Mg}$ & 1.811 & 1.992 & 1.988 & 2.103 & 2.147 \\
\hline $\mathrm{Cr}$ & 0.020 & - & - & - & - \\
\hline $\mathrm{V}$ & - & - & 0.024 & - & - \\
\hline$Y_{\text {total }}$ & 3.174 & 3.260 & 3.249 & 3.189 & 3.229 \\
\hline $\mathrm{Ca}$ & 0.190 & 0.109 & 0.113 & 0.271 & 0.265 \\
\hline $\mathrm{Na}$ & 0.000 & 0.000 & 0.000 & 0.000 & 0.000 \\
\hline$X_{\text {total }}$ & 0.190 & 0.109 & 0.113 & 0.271 & 0.265 \\
\hline [] & 0.810 & 0.891 & 0.887 & 0.729 & 0.735 \\
\hline Total & 15.364 & 15.369 & 15.362 & 15.460 & 15.494 \\
\hline $\mathrm{R} 1$ & 0.190 & 0.109 & 0.113 & 0.271 & 0.265 \\
\hline $\mathrm{R} 2$ & 2.795 & 2.928 & 2.932 & 2.930 & 2.974 \\
\hline $\mathrm{R} 1+\mathrm{R} 2$ & 2.985 & 3.037 & 3.045 & 3.201 & 3.239 \\
\hline R3 & 6.513 & 6.403 & 6.383 & 6.437 & 6.499 \\
\hline Mg\# & 0.648 & 0.680 & 0.678 & 0.718 & 0.722 \\
\hline
\end{tabular}

n.d. = not determined; $<$ d.l. = below detection limit. 
Excel worksheets and checked with Yavuz's (1997) Tourmal program, that assumes for $\mathrm{Fe}^{3+}$ calculation full occupancy of $\mathrm{T}$ and $\mathrm{Z}$ sites:

$\mathrm{T}=\mathrm{Si}+\mathrm{Al}^{\mathrm{IV}}=6.000$

$\mathrm{Z}=\mathrm{Al}^{\mathrm{IV}}+\mathrm{Fe}^{3+}=6.000$

Yavuz (1997) calculates the R1, R2 and R3 parameters as follows:

$$
\begin{aligned}
& \mathrm{R} 1=\mathrm{Na}+\mathrm{Ca} \\
& \mathrm{R} 2=\mathrm{Fe}_{\text {total }}+\mathrm{Mg} \\
& \mathrm{R} 3=\mathrm{Al}+1.33 \mathrm{Ti}
\end{aligned}
$$

Tourmaline compositions are represented in Figure 3, which is a Henry and Guidotti's (1985) Al-Fe tot $-\mathrm{Mg}$ diagram (molecular proportions). Tourmalines from metachert of Tapera Grande and Quartzito, and quartz veins of Tapera Grande fall on the line limiting field $2(\mathrm{Li}$ poor granitoids and their associated pegmatites and aplites), field 4 (metapelites and metapsammites coexisting with an Al-saturating phase) and field 5 (metapelites and metapsammites not coexisting with an Al-saturating phase), corresponding to intermediate schorl-dravite. Tourmalines of quartz veins from Quartzito fall in field 5 .

In the $\mathrm{Ca}-\mathrm{Fe}_{\text {tot }}-\mathrm{Mg}$ diagram (Fig. 4), tourmalines from metachert of Tapera Grande and Quartzito, tourmalinite and quartz veins of Tapera Grande fall in field 2 (Li-poor granitoids and their associated pegmatites and aplites) and 4 (Ca-poor metapelites, metapsammites, and quartz-tourmaline rocks), whereas tourmalines from quartz veins of Quartzito fall in field 4.

Differently from the other tourmalines, those from tourmalinite T12 show large $\mathrm{Al}_{50} \mathrm{Fe}(\text { total })_{50}$ and $\mathrm{Al}_{50} \mathrm{Mg}_{50}$ variation. These compositions are similar to those presented by Plimer (1988), which describes tourmalines from tourmalinites of eight different localities in Australia. The triangular diagrams also show that T12 tourmaline compositions are intermediate between those obtained for metachert and Quartzito quartz veins. Tourmaline compositions corresponding to Quartzito quartz veins match those found in Plimer (1986), Bone (1988), and King and Kerrich (1989).

Triangular diagrams of Figs. 3 and 4 show that $\mathrm{Mg}$ is a good discriminant factor that distinguishes Tapera Grande tourmalines from those of Quartzito quartz veins, i.e. the latter are $\mathrm{Mg}$ richer than the former.

It is worth mentioning that even when $\mathrm{K}_{2} \mathrm{O}$ (in all analyses) and $\mathrm{Na}_{2} \mathrm{O}$ (in tourmalines of Quartzito quartz veins) contents are below detection limits of EDS-SEM, yielding incomplete structural formulae, positive correlation between $\mathrm{Mg \#}\left[=\mathrm{Mg} /\left(\mathrm{Mg}+\mathrm{Fe}_{\text {total }}\right)\right]$ and $\mathrm{X}$-site vacancy $\left(\square_{\mathrm{X}}=1-\mathrm{Ca}-\mathrm{Na}-\mathrm{K}\right.$ ) is obtained, as depicted from Table I:

Metachert:

$\mathrm{Mg \#}=0.31-0.49 ; \quad \square_{\mathrm{X}}=0-0.24$

Tapera Grande quartz veins:

$\mathrm{Mg \#}=0.31-0.47 ; \quad \square_{\mathrm{X}}=0.04-0.21$

Tourmalinite:

$\mathrm{Mg} \#=0.25-0.62 ; \quad \square_{\mathrm{X}}=0.16-0.47$

Quartzito quartz veins:

$\mathrm{Mg \#}=0.61-0.68 ; \quad \square_{\mathrm{X}}=0.68-0.89$

Tourmalines of Quartzito quartz veins stand out not only for their higher $\mathrm{MgO}$ contents, and $\mathrm{NaO}$ contents below EDS-SEM detection, but also for the presence of $\mathrm{Cr}$ and sometimes V. Once Na results null in the structural formula and $\mathrm{Ca}$ is very low (0.11-0.21), $\mathrm{X}$-site vacancy reaches very high values $(0.68$ 0.89). On the other hand, $\mathrm{Mg}$ can be as high as 1.81-1.99, causing $\mathrm{Y}_{\text {total }}>3$; exceeding Al results in $\mathrm{Al}_{\mathrm{T}}$ from 0.07 to 0.19 (once $\mathrm{Si}<6$ ) and $\mathrm{Al}_{\mathrm{Y}}$ from 0.19 to 0.36 , accompanied by either 

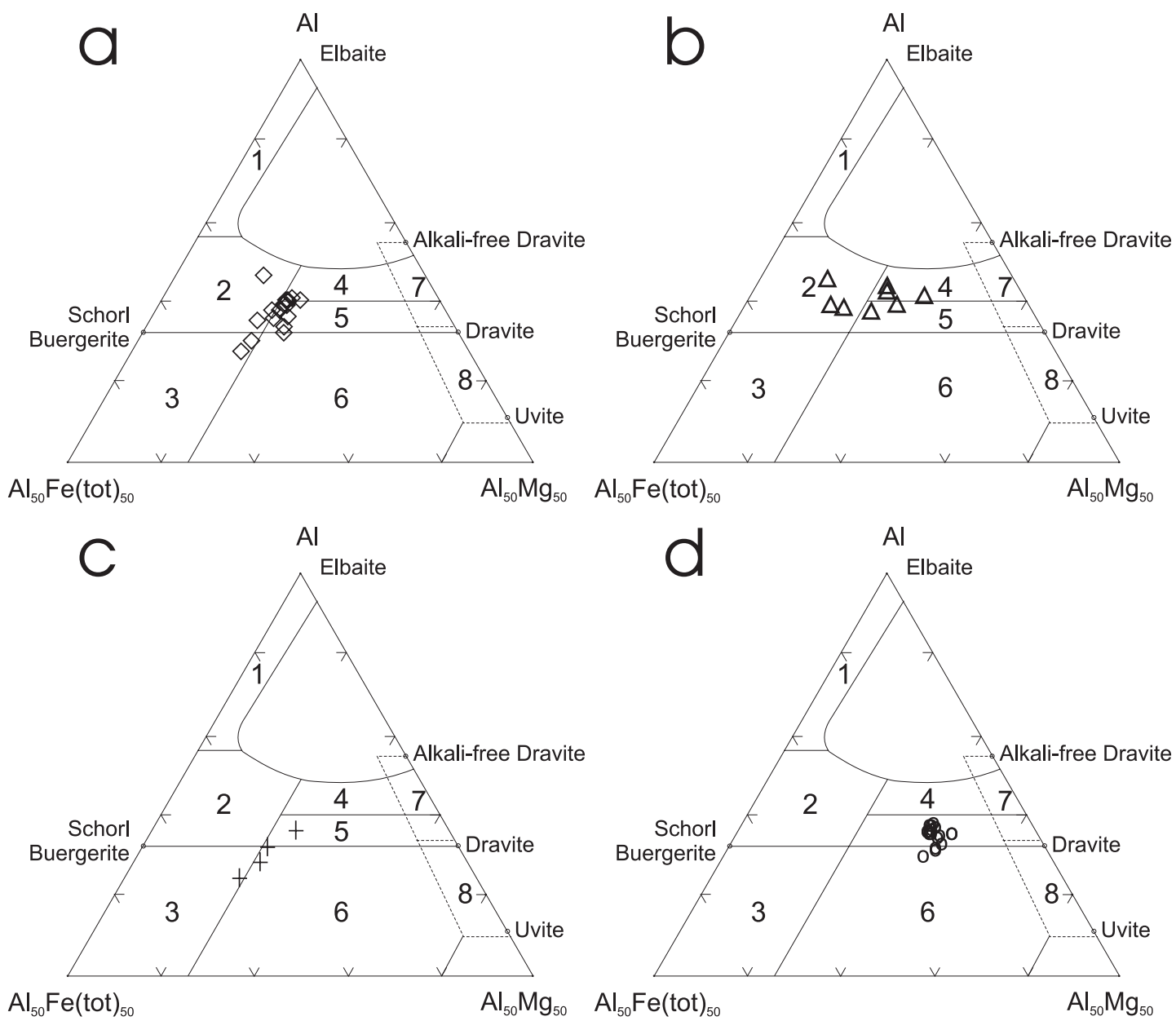

Fig. 3 - Representation of tourmaline compositions in Henry and Guidotti's (1985) Al-Fe tot $-\mathrm{Mg}$ diagram (molecular proportions). Tourmalines from: (a) metachert - Tapera Grande and Quartzito; (b) tourmalinite - Tapera Grande; (c) quartz vein - Tapera Grande, and (d) quartz vein - Quartzito. Fields: (1) Li-rich granitoid pegmatites and aplites; (2) Lipoor granitoids and their associated pegmatites and aplites; (3) $\mathrm{Fe}^{3+}$ rich quartz-tourmaline rocks (hydrothermally altered granites); (4) metapelites and metapsammites coexisting with an Al-saturating phase; (5) metapelites and metapsammites not coexisting with an $\mathrm{Al}$-saturating phase; (6) $\mathrm{Fe}^{3+}$ rich quartz-tourmaline rocks, calc-silicate rocks, and metapelites; (7) low-Ca metaultramafics and Cr, V-rich metasediments, and (8) metacarbonates and metapyroxenites.

Cr (0-0.04) or V (0-0.02).

R1+R2 vs. R3 diagrams (Fig. 5) show that tourmaline compositions from Quartzito quartz veins fall on the $\{\square \mathrm{Al}\}\{\mathrm{Na}[\mathrm{Fe}, \mathrm{Mg}]\}_{-1}$ vector, which represents the exchange schorl-dravite $\rightarrow$ foitite.

Slightly zoned crystals also illustrate the general behavior of Tapera Grande and Quartz- ito tourmaline compositions. Analyses 19, 20 and 21 in Table I correspond to points at rim, intermediate position and core of a LJ10A (metachert of Tapera Grande) tourmaline crystal (Fig. 2C). Numbers of Al and Mg apfu increase from point 1 to 2 and decrease to inner point 3, whereas $\mathrm{Fe}_{\text {total }}$ and $\mathrm{Na}$ apfu decrease from 1 to 2 followed by increase from 2 to 3 

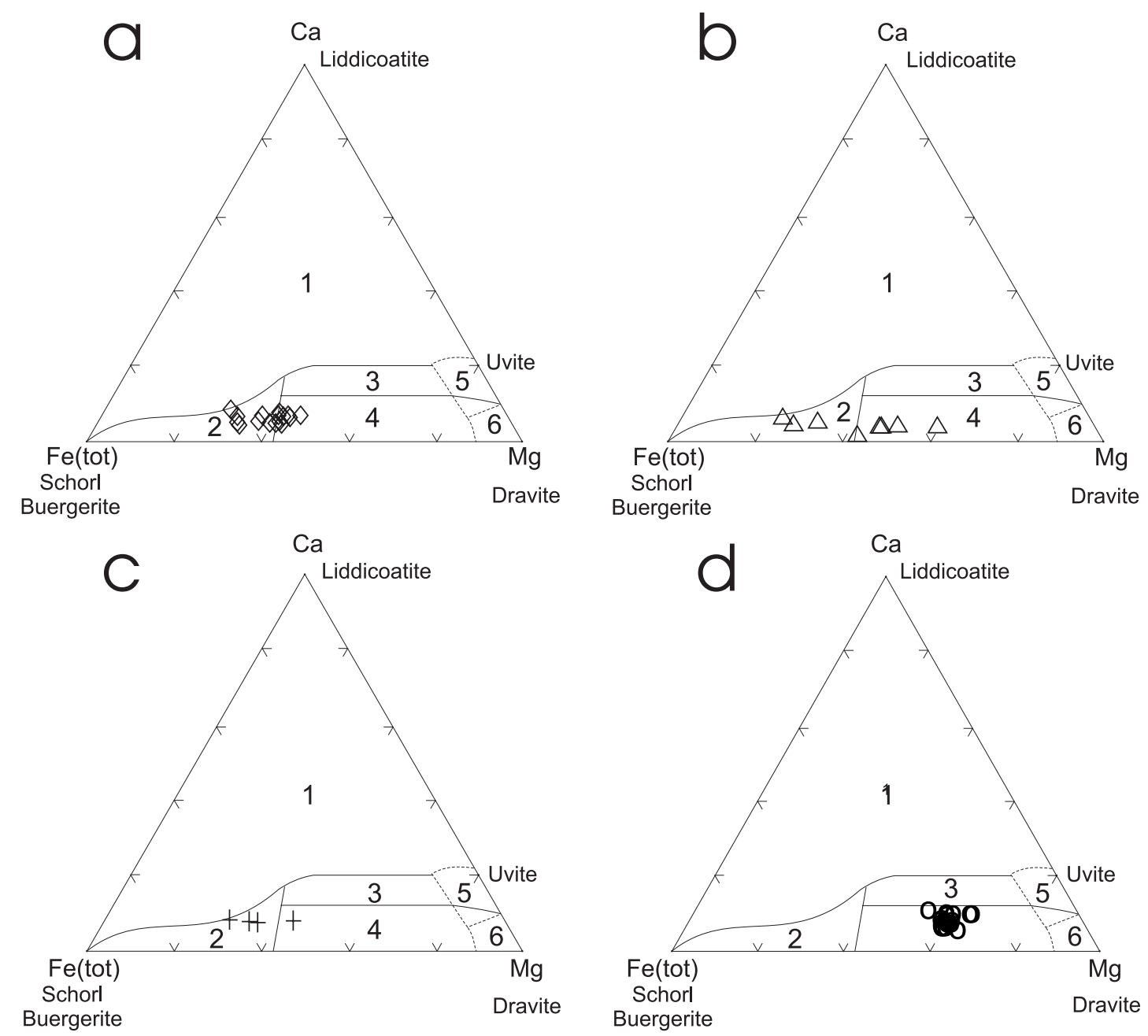

Fig. 4 - Representation of tourmaline compositions in Henry and Guidotti's (1985) Ca-Fetot-Mg diagram (molecular proportions). Tourmalines from: (a) metachert - Tapera Grande and Quartzito; (b) tourmalinite - Tapera Grande; (c) quartz vein - Tapera Grande, and (d) quartz vein - Quartzito. Fields: (1) Li-rich granitoid pegmatites and aplites; (2) Li-poor granitoids and their associated pegmatites and aplites; (3) Ca-rich metapelites, metapsammites, and calcsilicate rocks; (4) Ca-poor metapelites, metapsammites, and quartz-tourmaline rocks; (5) metacarbonates, and (6) metaultramafics.

(Fig. 6). The number of $\mathrm{Fe}_{\text {total }}$ apfu is higher than $\mathrm{Mg}$.

Zoned crystals of T12 tourmaline (analyses 23, 24 and 25 in Table I) show discrete decreasing $\mathrm{Mg}$ and $\mathrm{Na}$ apfu, and increasing $\mathrm{Fe}_{\text {total }}$ apfu from rim to core (Fig. 6), and slight $\mathrm{Al}$ apfu decrease and $\mathrm{Ti}$ and $\mathrm{Ca}$ apfu increase in an intermediate position between rim and core (analysis 24).

Figure 2D shows two zoned tourmaline needles of Quartzito quartz vein (FQ112-3b), which are also illustrated in Figure 2B, and the location of the points analyzed by EDS-SEM. Average values of analyses 63 and 64 (point 2, 

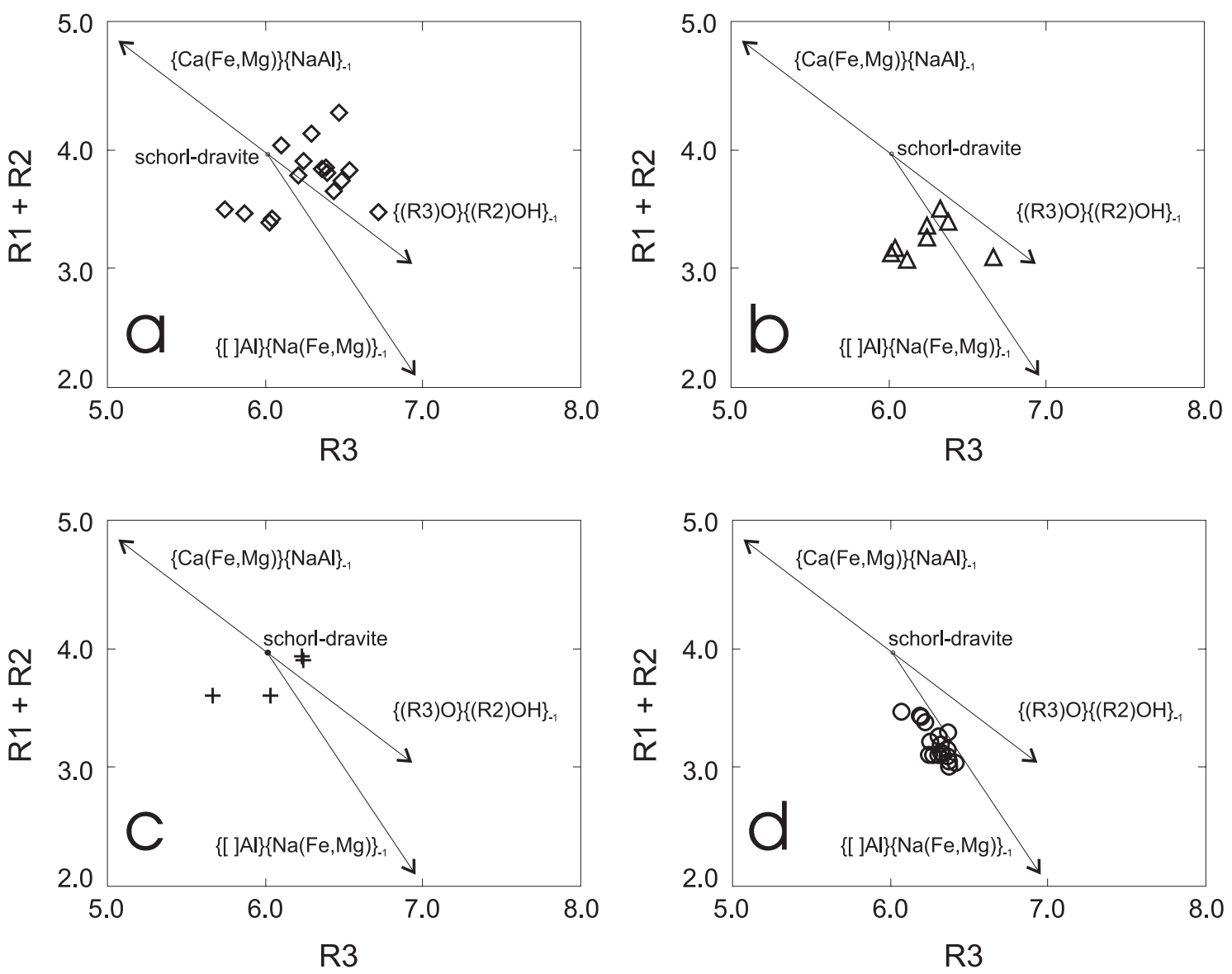

Fig. 5 - Representation of tourmaline compositions in the R1 + R2 vs. R3 diagram (R1, R2 and R3 calculated after Yavuz 1997). Tourmalines from: (a) metachert - Tapera Grande and Quartzito; (b) tourmalinite - Tapera Grande; (c) quartz vein - Tapera Grande, and (d) quartz vein - Quartzito.

at rim) and 67, 68 and 71 (point 3, at core) and analyses 72, 75 and 76 (point 4, at rim) and 79 and 80 (point 5, at core) are represented in Figure 6. The main compositional distinctions from rim to core are increase of $\mathrm{Si}$ and $\mathrm{Mg}$ apfu and decrease of $\mathrm{Al}$ and $\mathrm{Fe}_{\text {total }}$ apfu, and at the rims higher $\mathrm{Ti}, \mathrm{Fe}$ and $\mathrm{Ca}$ apfu. $\mathrm{Cr}$ seems to be higher at rims than at cores (Table I).

X-ray Fluorescence Analyses of Mineral SEPARATES

As stated by Yavuz (1997), normalization based on 31 atoms of oxygen causes the exaggera- tion of the relative importance of alkali-defect substitution. However, as previously stated, high X-site vacancies in tourmalines of Quartzito quartz veins resulted from $\mathrm{NaO}$ (and $\mathrm{K}_{2} \mathrm{O}$ ) contents below EDS-SEM detection limits, and relatively low $\mathrm{CaO}$ contents.

In order to check the actual X-site deficiency, tourmaline separates from tourmalinebearing quartz veins of Tapera Grande (SRT297 and SRT3-99) and Quartzito (FQ1123a) were analyzed by X-ray fluorescence. The analyses confirmed that the Quartzito samples are indeed alkali-deficient, $\mathrm{Cr}$ - and V-bearing 


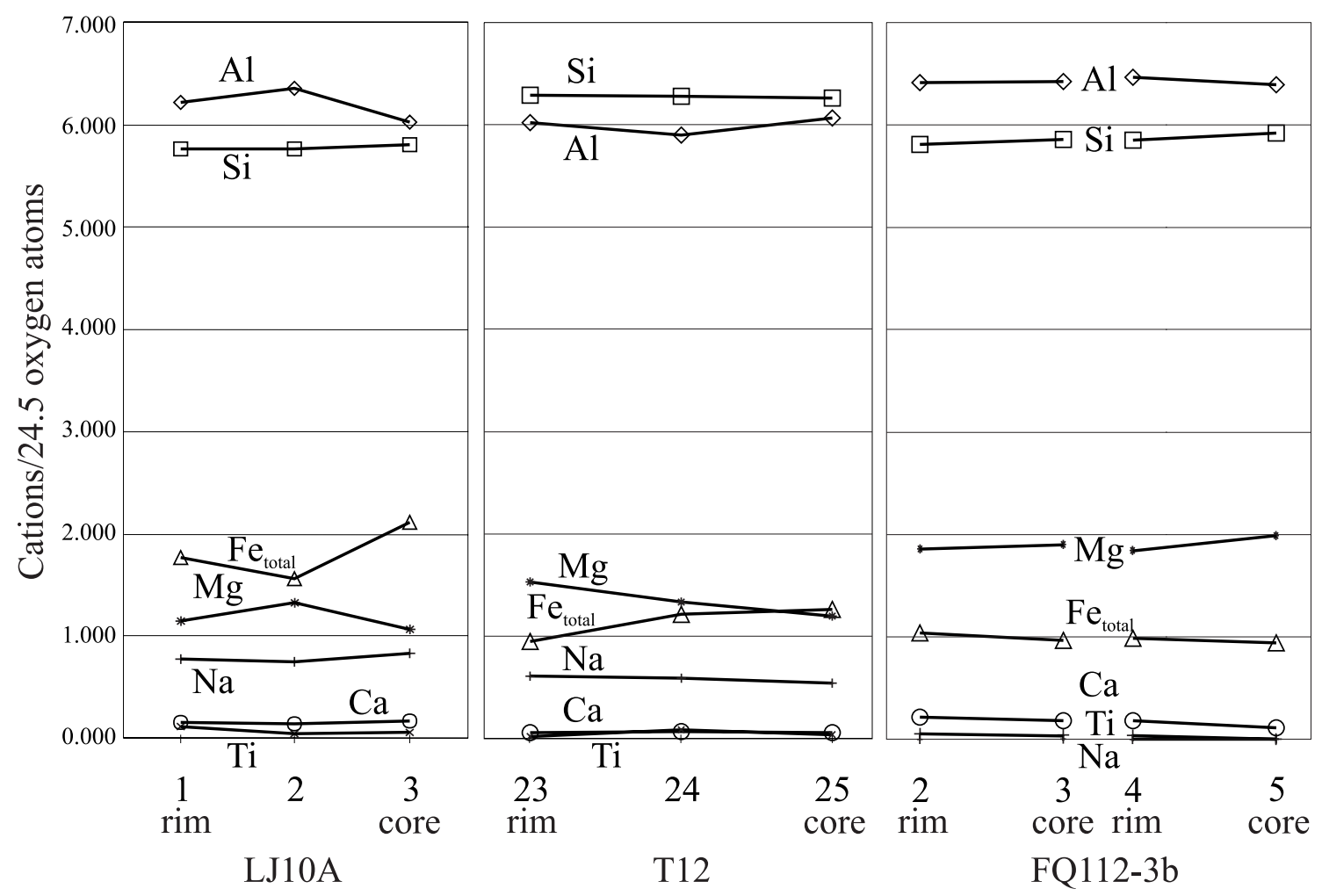

Fig. 6 - Variations of number of atoms per formula unit (apfu) from rim to core of tourmaline crystals in metachert (LJ10A - Tapera Grande), tourmalinite (T12 - Tapera Grande) and quartz vein (FQ112-3b - Quartzito).

tourmalines (Table II). The structural formula proposed for the FQ112-3a tourmaline is:

$$
\left[\square_{0.51} \mathrm{Ca}_{0.33} \mathrm{Na}_{0.15}\right]
$$

$\left[\mathrm{Ti}_{0.07} \mathrm{Fe}_{1.07} \mathrm{Cr}_{0.01} \mathrm{Ni}_{0.01} \mathrm{~V}_{0.01} \mathrm{Mg}_{1.79} \mathrm{Si}_{0.03}\right]$

$$
\left[\mathrm{Al}_{5.98} \mathrm{Fe}_{0.02}\right] \mathrm{Si}_{6} \mathrm{O}_{18}\left(\mathrm{BO}_{3}\right)_{3}(\mathrm{OH})_{4}
$$

which corresponds to an intermediate composition between foitite (McDonald et al. 1993) and magnesiofoitite (Hawthorne et al. 1999).

\section{RAMAN SPECTROSCOPY}

Polished thin sections of tourmaline-rich metachert (LJ10) and tourmalinite (T12) from Tapera Grande, and a tourmaline-rich quartz vein (FQ-112-3a) from Quartzito were analyzed by Raman spectrometry. Raman spectra of schorl GRR\#794 from Cahuilla Mountain (Riverside,
California) and a magnesiofoitite from Honshu (Japan) - available at the Mineral Spectroscopy Server of the California Institute of Technology (http://minerals.gps.caltech.edu) - are presented for comparison.

Peaks corresponding to tourmalines from LJ10 and T12 appear at 235-239 $\mathrm{cm}^{-1}, 360-$ $367 \mathrm{~cm}^{-1}$, and $635-700 \mathrm{~cm}^{-1}$ intervals (three consecutive peaks - Fig. 7.2a). According to Gasharova et al. (1997), these peaks are characteristic of G1 - intermediate between buergerite and schorl of the buergerite-schorl group. For tourmaline T12, peaks at 3228 and $3475 \mathrm{~cm}^{-1}$ were obtained (Fig. 7.2b), the former having no correspondence with schorl GRR\#794 spectrum close to the $\mathrm{OH}$ region $\left(3400-3800 \mathrm{~cm}^{-1}\right.$ interval - Fig. 7.1b). 


\section{TABLE II}

XRF analyses of tourmalines from Tapera Grande and Quartzito. Structural formulae calculated on 31 oxygen basis.

\begin{tabular}{|c|c|c|c|c|c|c|c|}
\hline Sample & SRT-2-97 & SRT-3-99 & FQ112-3A & Sample & SRT-2-97 & SRT-3-99 & FQ112-3A \\
\hline $\mathrm{SiO}_{2}$ & 35.97 & 35.42 & 36.71 & $\mathrm{~B}$ & 3.000 & 3.000 & 3.000 \\
\hline $\mathrm{TiO}_{2}$ & 0.90 & 0.69 & 0.56 & & & & \\
\hline $\mathrm{Al}_{2} \mathrm{O}_{3}$ & 28.84 & 29.10 & 30.84 & $\mathrm{Al}_{\text {total }}$ & 5.738 & 5.813 & 5.977 \\
\hline $\mathrm{Cr}_{2} \mathrm{O}_{3}$ & 0.00 & 0.00 & 0.08 & $\mathrm{Si}_{\text {total }}$ & 6.070 & 6.002 & 6.035 \\
\hline $\mathrm{FeO}$ & 13.35 & 13.81 & 7.95 & & & & \\
\hline $\mathrm{MnO}$ & 0.03 & 0.03 & 0.00 & $\mathrm{Si}_{\mathrm{T}}$ & 6.000 & 6.000 & 6.000 \\
\hline $\mathrm{MgO}$ & 4.22 & 4.18 & 7.31 & $\mathrm{Al}_{\mathrm{T}}$ & 0.000 & 0.000 & 0.000 \\
\hline $\mathrm{CaO}$ & 1.79 & 1.48 & 1.89 & & & & \\
\hline $\mathrm{Na}_{2} \mathrm{O}$ & 1.44 & 1.78 & 0.47 & $\mathrm{Al}_{\mathrm{Z}}$ & 5.738 & 5.813 & 5.977 \\
\hline $\mathrm{K}_{2} \mathrm{O}$ & 0.14 & 0.20 & 0.01 & $\mathrm{Fe}^{3+}$ & 0.262 & 0.187 & 0.023 \\
\hline $\mathrm{V}_{2} \mathrm{O}_{5}$ & 0.02 & 0.04 & 0.09 & & & & \\
\hline \multirow[t]{3}{*}{$\mathrm{NiO}$} & $<$ d.1. & $<$ d.1. & 0.09 & $\mathrm{Al}_{\mathrm{Y}}$ & 0.000 & 0.000 & 0.000 \\
\hline & & & & $\mathrm{Si}_{Y}$ & 0.070 & 0.002 & 0.035 \\
\hline & ppm & $\mathrm{ppm}$ & ppm & $\mathrm{Ti}$ & 0.114 & 0.087 & 0.070 \\
\hline $\mathrm{Ba}$ & $<37$ & $<37$ & 62 & $\mathrm{Fe}_{\text {total }}$ & 1.885 & 1.957 & 1.092 \\
\hline $\mathrm{Ce}$ & 215 & 280 & 95 & $\mathrm{Fe}^{2+}$ & 1.622 & 1.770 & 1.070 \\
\hline Co & 29 & 28 & 44 & $\mathrm{Cr}$ & 0.000 & 0.000 & 0.010 \\
\hline $\mathrm{Cr}$ & $<13$ & $<13$ & 534 & $\mathrm{Ni}$ & 0.000 & 0.000 & 0.012 \\
\hline $\mathrm{Cu}$ & 71 & 344 & 2323 & $\mathrm{~V}$ & 0.001 & 0.003 & 0.006 \\
\hline $\mathrm{Ga}$ & 59 & 65 & 45 & $\mathrm{Mn}$ & 0.004 & 0.004 & 0.000 \\
\hline $\mathrm{La}$ & $<28$ & $<28$ & 64 & $\mathrm{Mg}$ & 1.061 & 1.056 & 1.791 \\
\hline $\mathrm{Nb}$ & 38 & 27 & 85 & $Y_{\text {total }}$ & 2.873 & 2.922 & 2.993 \\
\hline $\mathrm{Nd}$ & 160 & 38 & 33 & & & & \\
\hline $\mathrm{Ni}$ & $<3$ & $<3$ & 705 & $\mathrm{Ca}$ & 0.324 & 0.269 & 0.333 \\
\hline $\mathrm{Pb}$ & $<3$ & $<3$ & $<3$ & $\mathrm{Na}$ & 0.471 & 0.585 & 0.150 \\
\hline $\mathrm{Rb}$ & 18 & 8 & 29 & $\mathrm{~K}$ & 0.030 & 0.043 & 0.001 \\
\hline$S$ & 61 & 705 & 3207 & $\mathrm{X}_{\text {total }}$ & 0.825 & 0.897 & 0.484 \\
\hline $\mathrm{Sc}$ & $<14$ & 77 & 44 & [] & 0.175 & 0.103 & 0.516 \\
\hline $\mathrm{Sr}$ & 497 & 444 & 489 & & & & \\
\hline Th & $<6$ & $<6$ & $<6$ & Total Cations & 15.698 & 15.819 & 15.477 \\
\hline $\mathrm{U}$ & 37 & 33 & 62 & & & & \\
\hline V & 138 & 211 & 488 & $\mathrm{R} 1$ & 0.795 & 0.854 & 0.483 \\
\hline Y & 127 & 57 & 55 & $\mathrm{R} 2$ & 2.946 & 3.013 & 2.883 \\
\hline $\mathrm{Zn}$ & 154 & 111 & $<2$ & R3 & 5.889 & 5.929 & 6.070 \\
\hline $\mathrm{Zr}$ & 459 & 130 & 232 & Mg\# & 0.360 & 0.350 & 0.621 \\
\hline
\end{tabular}


TABLE III

\section{Stable oxygen and hydrogen isotope analyses for quartz and tourmalines from Tapera Grande and Quartzito.}

\begin{tabular}{l|c|c|c|c}
\hline Sample & & $\begin{array}{c}\delta^{18} \text { OSMOw quartz }_{\text {(per mil) }} \\
\text { LF-10B }\end{array}$ & $\begin{array}{c}\delta^{18} \text { OSMOw tourmaline }_{\text {per mil) }} \\
\text { LJ-09 }\end{array}$ & $\begin{array}{c}\delta \text { D tourmaline } \\
\text { (per mil) }\end{array}$ \\
SRT-2-96B & metachert & 13.9 & 12.9 & -104 \\
SRT-3-99 & quartz vein & 13.2 & 13.5 & -103 \\
F-01(1B)-3,80m & quartz vein & 12 & 11.6 & -86 \\
F-04-17,55m & metachert & 12.8 & 12 & \\
FQ-112-(3A)-18,40m & metachert & 12.7 & 11.6 & -102 \\
\hline
\end{tabular}

Alkali-deficient tourmalines from Quartzito show peaks at $116,214,238,365,491$, $698-702,841 \mathrm{~cm}^{-1}$ in the $100-800 \mathrm{~cm}^{-1}$ interval (Fig. 7.4a), corresponding to G3V of the dravite-buergerite-uvite series of Gasharova et al. (1997), characterized by $0.30 \square_{\mathrm{X}}$ and $0.28 \mathrm{Ti}$ apfu. The peak found at $3578 \mathrm{~cm}^{-1}$ (Fig. 7.4b) is more likely coincident with schorl GRR\#794 peaks in the $\mathrm{OH}$ region than with the peaks at 3632 and $3622 \mathrm{~cm}^{-1}$ presented by Béziat et al. (1999) as indication of vacancy at the X-site, but more refined work has to be carried out.

Peaks characteristic of the elbaite group (at $224 \mathrm{~cm}^{-1}$, and at positions further than $707 \mathrm{~cm}^{-1}$ ) were not observed in the spectrograms.

\section{IsOTOPIC DATA}

Table III presents $\delta^{18} \mathrm{O}$ values obtained for quartz and tourmaline crystals from tourmalinerich metachert and quartz veins, and corresponding $\delta \mathrm{D}$ values for tourmalines.

The values of $\delta^{18} \mathrm{O}$ and $\delta \mathrm{D}$ for tourmalines are plotted in Figure 8, which shows that tourmalines from Tapera Grande and Quartzito have isotopic signatures different from those for primary magmatic waters and igneous rocks, being closer to the compositions that correspond to sediment waters.

The values of $\delta^{18} \mathrm{O}$ for quartz and tourmalines from tourmaline-rich quartz veins and metachert are similar, the isotope fractionation between the two minerals varying from 0 to 1.8 per mil. Entering these values in Kotzer's et al. (1993) empirical oxygen-isotope fractionation factor equation, an oxygen-isotope equilibration temperature of $580^{\circ} \mathrm{C}$ is obtained.

\section{DISCUSSION}

A wide range of tourmaline compositions is identified in the Morro da Pedra Preta Formation, from intermediate schorl-dravite with varying $\mathrm{Al}$ contents (tourmalines from metachert and quartz veins), intermediate schorldravite with varying $\mathrm{Mg}$ and $\mathrm{Fe}$ contents (tourmalines from tourmalinite), and $\mathrm{Cr}(-\mathrm{V})$-bearing foitite-magnesiofoitite, which is characterized by high $\mathrm{Mg}$ contents and X-site deficiency (Quartzito quartz veins). Discrete variations of $\mathrm{Mg}, \mathrm{Fe}_{\text {total }}, \mathrm{Al}$ and $\mathrm{Ca}$ apfu are observed 


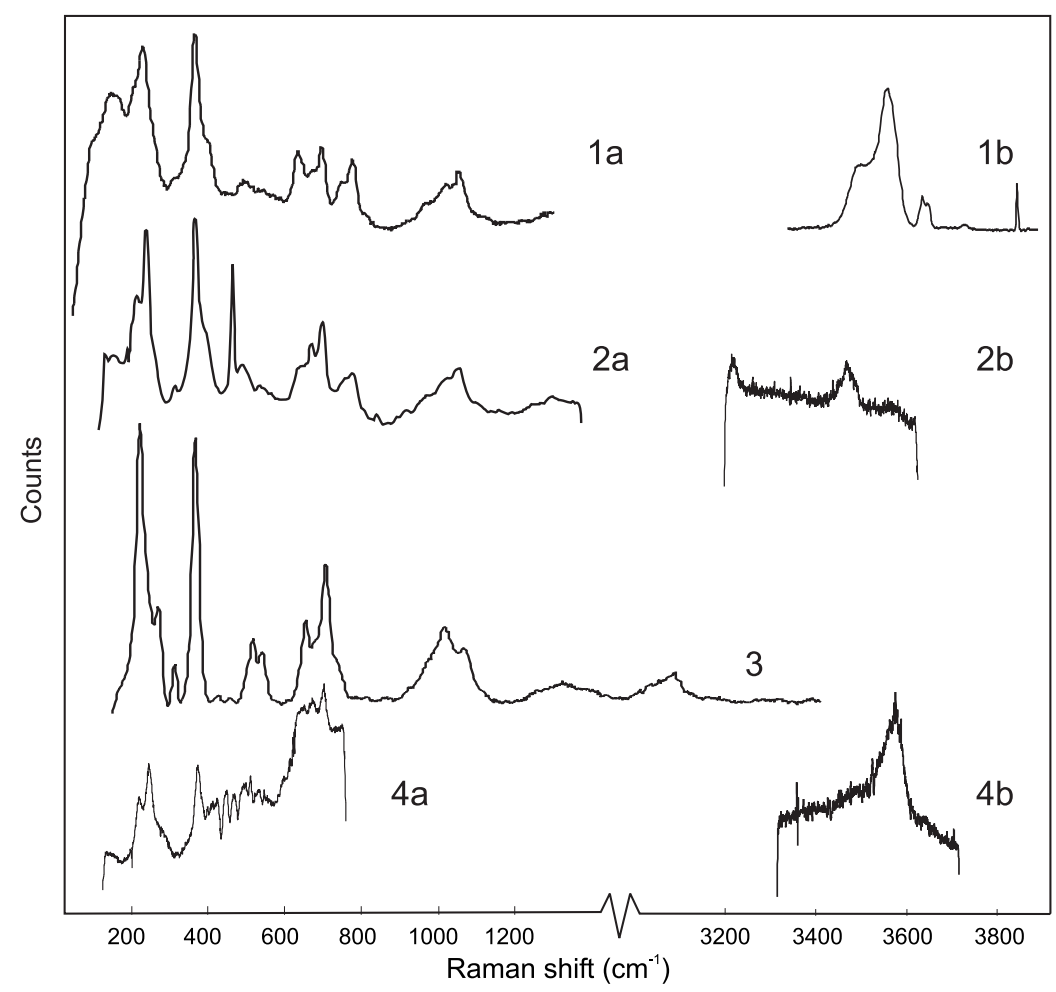

Fig. 7 - Raman spectra of (1) schorl GRR\#794 from Cahuilla Mountain, Riverside (California); (2) tourmalines from metachert LJ10 and tourmalinite T12 (Tapera Grande); (3) magnesiofoitite from Honshu (Japan), and (4) tourmaline from quartz vein FQ112-3a (Quartzito).

\section{TABLE IV}

XRF analyses ( $\mathrm{Cr}$ and $\mathrm{V}$ ) of host rocks of Quartzito quartz veins.

\begin{tabular}{l|c|c|c}
\hline Sample & Depth & Cr (ppm) & V (ppm) \\
\hline FQ112-2 & 17.90 to $18.80 \mathrm{~m}$ & 205 & 233 \\
FQ112-43-1 & 43.45 to $43.40 \mathrm{~m}$ & 76 & 235 \\
FQ-112 & 44.00 to $44.80 \mathrm{~m}$ & 557 & 191 \\
F112-45-4 & 45.43 to $45.58 \mathrm{~m}$ & 714 & 234 \\
FQ112-47 $(\mathrm{MgO}=15.96 \%)$ & $47 \mathrm{~m}$ & 74 & 136 \\
FQ-112-48-1 & 48.15 to $48.30 \mathrm{~m}$ & 711 & 224 \\
\hline
\end{tabular}

in zoned crystals of intermediate schorl-dravite and foitite-magnesiofoitite, as well as variations of $\mathrm{Na}$ and $\mathrm{Ti}$ in the former and $\mathrm{Cr}$ (and $\mathrm{V}$ ) in the latter.

Compositional variations in growing tourmalines can be related to variations in environ- 


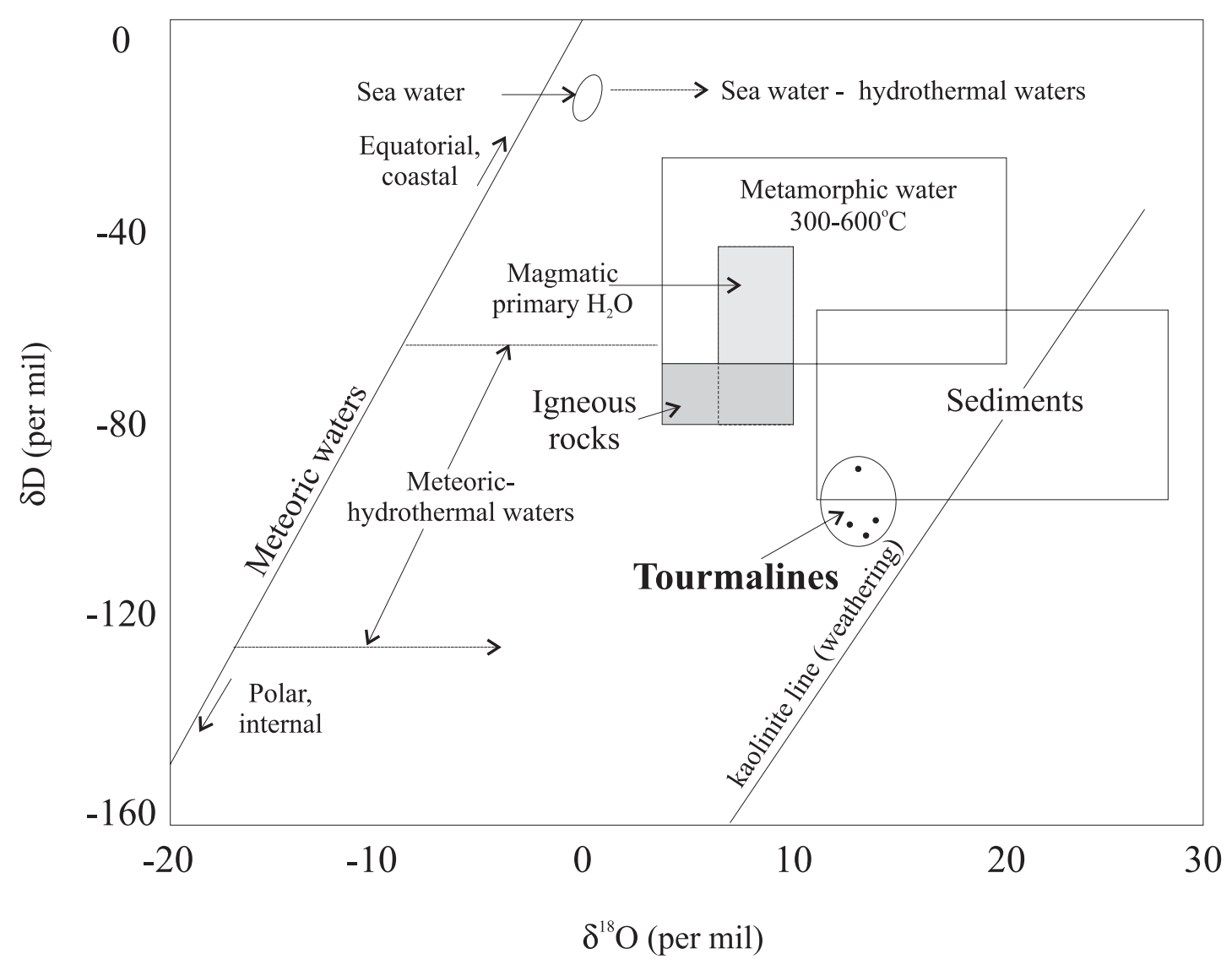

Fig. 8 - Representation of $\delta^{18} \mathrm{O}$ and $\delta \mathrm{D}$ values obtained for tourmalines from Tapera Grande and Quartzito in Taylor's (1967) diagram for isotopic compositions of waters from several origins.

ment and metamorphic conditions and composition of circulating fluids, but once tourmaline is formed, it is stable under a wide range of physical-chemical conditions. Henry and Dutrow (1996) report the occurrence of tourmalines according to increasing metamorphic grades, showing that there is a correlation between $\mathrm{Al}, \mathrm{Ca}$ and $\mathrm{X}$-site-vacancy contents that apparently does not depend on metamorphism, but rather on the phases present. For example, in metapelites, aluminous, high-X-site-vacancy tourmalines coexist with aluminous minerals such as staurolite and sillimanite; there is an inverse correlation between $\mathrm{Ca}$ and $\mathrm{X}$-site vacancy. On the other hand, tourmalines that grow in low-Al metapelites contain lower values of $\mathrm{X}$-site vacancy. In medium-grade metapelites and quartzites, tourmalines are intermediate schorl-dravite with variable $\mathrm{Al}$ and $\mathrm{X}$-sitevacancy amounts. Although in this study tourmalines closely related to metamorphic minerals (such as those found in basic metatuffs and volcanic rocks) have not been analyzed, a certain correlation between $\mathrm{Al}$ and $\mathrm{X}$-site vacancy contents is observed for the studied tourmalines from Tapera Grande and Quartzito.

Henry and Dutrow (1996) also stated that metamorphosed stratiform tourmalinites, typically associated with metapelites, metapsammites or meta-ironstones, are interpreted as be- 
ing formed by early diagenesis of B-rich chemical precipitates, or by metasomatic modifications due to volcanic exhalations during sedimentation. Tourmalines from stratiform tourmalinites have several common chemical characteristics with tourmalines developed in metapsammites and metapelites, e.g., they can be peraluminous, coexisting with Al-saturated minerals such as sillimanite, or can be less aluminous if they coexist with less aluminous minerals. These findings agree with compositional variations obtained for Tapera Grande T12 tourmalinite.

Tourmaline, developed as a consequence of infiltration of B-bearing hydrothermal fluids, can have a wide range of compositions that depend on the compositions of the altering host rock and the invading fluid. This type of $\mathrm{B}$ metasomatism is commonly associated with a wide variety of precious metal deposits such as $\mathrm{Au}, \mathrm{Ag}, \mathrm{Zn}, \mathrm{U}$, and Mo. In the Quartzito area, scheelite, molybdenite, and sphalerite were identified in drillhole samples, associated with pyrite and chalcopyrite.

The tourmaline chemistry is dominated by the nature of the host rock, but in other cases tourmaline has a "mixed" chemical signature influenced by the chemistry of the fluids. When hydrothermal fluid becomes dominant, the Brich fluids can lead to extensive tourmalinization. It is relatively selective in that B-rich fluids will preferentially tourmalinize rocks with the requisite $\mathrm{Mg}-\mathrm{Fe}-\mathrm{Al}$ constituents. In general these tourmalines tend to have compositions of schorl to intermediate schorl-dravite, but can exhibit considerable amount of $\mathrm{X}$-site vacancy and substitution of $\mathrm{Fe}^{3+}$ (e.g. Cavarretta and Puxeddu 1990). This is the case of Quartzito tourmaline-bearing quartz veins, whose compositions were also affected by the host rock chem- istry. In Table IV $\mathrm{Cr}$ and $\mathrm{V}$ contents obtained from XRF analyses of host rocks of Quartzito quartz veins are listed. Regarding $\mathrm{Cr}$, the values obtained for Quartzito are, at present, the highest ones found in whole rock analyses of drillhole samples of Tapera Grande and Quartzito. High V contents are found in a variety of rocks, especially those of sedimentary origin.

\section{CONCLUSIONS}

From the data here presented, it is concluded that:

- Tourmalines from Tapera Grande and Quartzito metachert and from Tapera Grande tourmalinite are intermediate schorldravite, of syngenetic origin, formed under submarine, sedimentary-exhalative conditions. Amphibolite-grade metamorphism did not strongly affect their compositions.

- Quartz veins that crosscut the Morro da Pedra Preta Formation not only contain intermediate schorl-dravite (Tapera Grande), but also alkali-deficient, Cr-(V-)bearing foitite-magnesiofoitite (Quartzito), which are characterized by higher Mg\# values than those for intermediate schorl-dravite.

- Foitite-magnesiofoitite reflects the composition of the host rocks of Quartzito quartz veins, where fluid percolation along faults and fractures associated with the Sertãozinho Fault led to leaching of $\mathrm{Cr}$ (and V), and the crystallization of alkali-deficient, $\mathrm{Cr}-(\mathrm{V}-)$ bearing tourmalines together with quartz.

- Raman spectroscopy also confirms the existence two distinct groups of tourmalines in Tapera Grande and Quartzito areas, and rules out the presence of elbaite. Should 
magmatic/metasomatic origin be the case, schorlitic-elbaitic ( $\mathrm{Li}$ and $\mathrm{Al}$ rich) compositions would be expected, according to e.g. Plimer (1986).

- Stable isotope data indicate sediments as fluid sources for the intermediate schorldravite and foitite-magnesiofoitite, ruling out a direct (post-)magmatic origin. $\delta^{18} \mathrm{O}$ compositions for tourmalines and host metachert or quartz veins are very similar, suggesting that fluid equilibration occurred during crystallization of both minerals.

- In spite of the occurrence of granitic bodies intruding the Serra do Itaberaba Group, the studied tourmalines are not directly related to them. The same conclusion was drawn by Kassoli-Fournaraki and Michailidis (1994), when studying tourmalinebearing quartz veins crosscutting metamorphic rocks in Macedonia (northern Greece). There is still the possibility, though, that granitic bodies could have been the heat source for circulating, reactive fluids.

\section{ACKNOWLEDGMENTS}

The authors wish to thank Prof. Anthony E. Fallick for making stable oxygen and hydrogen isotope analyses at the Scottish Universities Environmental Research Center (East Kilbride, UK) possible, and to São Paulo State Research Foundation FAPESP (Process numbers 98/05526-09 and 99/05792-03) for financial support. The authors are indebted to the anonymous referees for their helpful suggestions.

\section{RESUMO}

Na Formação Morro da Pedra Preta, sequiência vulcano-sedimentar do Grupo Serra do Itaberaba
(São Paulo, Brasil), turmalinas de composição intermediária schorl-dravita ocorrem em formação ferrífera (incluindo turmalinito e metachert), metassedimentos, rochas cálcio-silicáticas e metabásicas a intermediárias.

A Formação Morro da Pedra Preta é cortada por veios de quartzo que contêm tanto schorl-dravita intermediária, como turmalinas com $\mathrm{Cr}$ e $\mathrm{V}$ deficientes em álcalis. A ocupação do sítio $\mathrm{X}$ é $\square_{0.51} \mathrm{Ca}_{0.33} \mathrm{Na}_{0.15}$, caracterizando-as como intermediárias aos membros extremos foitita e magnesiofoitita. Seus valores de Mg\# são mais elevados do que aqueles da schorl-dravita intermediária.

A espectroscopia Raman também confirma a presença de dois grupos de turmalinas. Dados de isótopos estáveis indicam como fontes de fluídos águas de origem sedimentar, em detrimento a fluídos oriundos de fonte (pós-)magmática. As composições de delta ${ }^{18} \mathrm{O}$ das turmalinas e quartzo dos veios são muito semelhantes, mostrando ter havido equilíbrio isotópico de fluídos durante sua cristalização.

Schorl-dravita intermediária formou-se em condições sedimentares-exalativas submarinas; sua composição não foi fortemente afetada pelo metamorfismo. Turmalinas mais jovens, de composição entre foitita-magnesiofoitita, refletem a composição das rochas hospedeiras dos veios de quartzo, sendo que os fluídos que percolaram falhas e fraturas lixiviaram $\mathrm{Cr}$ (e V), ocorrendo cristalização de turmalinas deficientes em álcalis com $\mathrm{Cr}$ (e V) em veios.

Palavras-chave: série schorlita-dravita, foititamagnesiofoitita, Grupo Serra do Itaberaba, Formação Morro da Pedra Preta.

\section{REFERENCES}

Beljavskis P, Juliani C, Garda GM, Xavier RP AND Bettencourt JS. 1999a. Overview 
of the gold mineralization in the metavolcanicsedimentary sequence of the Serra do Itaberaba Group - São Paulo - Brazil. In: Stanley CJ ET AL. (Ed.), Mineral Deposits: Processes to Processing, v.1, Rotterdam/Brookfield: Balkema, p. 151-153.

BelJavskis P, Garda GM and SAYeg IJ. 1999b. Application of SEM in the study of gold mineralizations in the Morro da Pedra Preta Formation, Grupo Serra do Itaberaba - São Paulo, Brazil. Acta Microscopica 8 (Supplement A): 125-126.

Béziat D, Bourges F, Debat P, Fuchs Y, Lompo M, Martin F, NikiÉma S and TolLON F. 1999. The Guibaré and Fété Kolé goldbearing tourmaline-quartz veins in the Birimian Greenstone belts of Burkina Faso. Canadian Mineralogist 37: 575-591.

Bone Y. 1988. The geological setting of tourmalinite at Rum Jungle, N.T., Australia - genetic and economic implications. Mineral Deposita 23: 34-41.

Cavarretta G and Puxeddu M. 1990. Schorldravite-ferridravite tourmalines deposited by hydrothermal magmatic fluids during early evolution of the Larderello geothermal field, Italy. Econ Geol 85: 1236-1251.

Fallick AE, McConville P, Boyce AJ, Burgess R and Kelley SP. 1992. Laser microprobe stable isotope measurements on geological materials: some experimental considerations (with special reference to $\delta^{34} \mathrm{~S}$ in sulphides). Chem Geol 101: 53-61.

Garda GM, Beljavskis P and Sayeg IJ. 1999. Gold mineralization in the Quartzito area, the Serra do Itaberaba Group - São Paulo, Brazil. Acta Microscopica 8 (Supplement A): 127-128.

Garda GM, Beljavskis P, Juliani C and Boyce AJ. 2002. Sulfur stable isotope signatures of the Morro da Pedra Preta Formation, Serra do Itaberaba Group, São Paulo State, Brazil. Geochimica Brasiliensis (in press).

Gasharova B, Mihailova B and Konstantinov L. 1997. Raman spectra of various types of tour- maline. Eur J Mineral 9: 935-940.

Hawthorne FC and Henry DJ. 1999. Classification of the minerals of the tourmaline group. Eur J Mineral 11: 201-215.

Hawthorne FC, Selway JB, Kato A, Matsubara S, Shimizu M, Grice, JD and Vajdak J. 1999. Magnesiofoitite,

[ ] $\left(\mathrm{Mg}_{2} \mathrm{Al}\right) \mathrm{Al}_{6}\left(\mathrm{Si}_{6} \mathrm{O}_{18}\right)\left(\mathrm{BO}_{3}\right)_{3}(\mathrm{OH})_{4}$, a new alkali-deficient tourmaline. Can Mineral 37: 1439-1443.

Henry DJ AND Dutrow BL. 1996. Metamorphic tourmaline and its petrologic applications. In: Grew ES AND ANovitz LM (Ed.), Boron Mineralogy, petrology and geochemistry. Rev Mineral 33: 503-557.

Henry DJ AND Guidotti CV. 1985. Tourmaline as a petrogenetic indicator mineral: an example from the staurolite-grade metapelites of NW Maine. Am Mineral 70: 1-15.

JANASI V AND UlbRICH HHGJ. 1991. Late Proterozoic granitoid magmatism in the state of São Paulo, southeastern Brazil. Precambrian Res 51: 351-374.

Juliani C. 1993. Geologia, petrogênese e aspectos metalogenéticos dos grupos Serra do Itaberaba e São Roque na região das Serras do Itaberaba e da Pedra Branca, NE da cidade de São Paulo, SP. São Paulo, 684p. (Doctorate Thesis. Instituto de Geociências da USP).

Juliani C AND Beljavskis P. 1995. Revisão da litoestratigrafia da faixa São Roque/Serra do Itaberaba (SP). Revista do IG 16(1/2): 33-58.

Juliani C, Hackspacker P, Dantas EL and FetTER AH. 2000. The Mesoproterozoic volcanosedimentary Serra do Itaberaba Group of the central Ribeira Belt, São Paulo State, Brazil: implications for the age of the overlying São Roque Group. Rev Bras Geoc 30: 82-86.

Kassoli-Fournaraki A AND MichaIlidis K. 1994. Chemical composition of tourmaline in quartz veins from Nea Roda and Thasos areas in Macedonia, Northern Greece. Can Mineral 32: 607-615. 
KIng RW and Kerrich R. 1989. Chromian dravite associated with ultramafic-rock-hosted Archean lode gold deposits, Timmins-Porcupine District, Ontario. Can Mineral 27: 419-426.

Kotzer TG, Kyser TK, King RW and Kerrich R. 1993. An empirical oxygen- and hydrogenisotope geothermometer for quartz-tourmaline and tourmaline-water. Geochim Cosmochim Acta 57: 3421-3426.

McDonald DJ, Hawthorne FC and Grice JD. 1993. Foitite, $\square\left[\left(\mathrm{Fe}_{2}^{2+}\left(\mathrm{Al}, \mathrm{Fe}^{3+}\right)\right] \mathrm{Al}_{6} \mathrm{Si}_{6} \mathrm{O}_{18}\left(\mathrm{BO}_{3}\right)_{3}(\mathrm{OH})_{4}\right.$, a new alkali-deficient tourmaline: Description and crystal structure. Am Mineral 78: 1299 1303.

Plimer IR. 1983. The association of tourmalinebearing rocks with mineralization at Broken Hill, N.S.W. Proc Ann Aus Inst Min Met Conf, p. $157-176$.

PLIMER IR. 1986. Tourmalinites from the Golden Dyke Dome, Northern Australia. Mineral Deposita 21: 263-270.

Plimer IR. 1987. The association of tourmaline with stratiform scheelite deposits. Mineral Deposita 22: 282-291.

Plimer IR. 1988. Tourmalines associated with Australian Proterozoic submarine exhalative ores. In: Friedrich GH AND Herzig PM. (Eds.) Base metal sulfide deposits in sedimentary and volcanic environments. Berlin: Springer-Verlag, p. 255-283.
SLACK JF. 1982. Tourmaline in AppalachianCaledonian massive sulphide deposits and its exploration significance. Trans Instn Min Metall 91: B81-B89.

SLACK JF. 1996. Tourmaline associations with hydrothermal ore deposits. In: GREw ES AND ANovitz LM (Ed.), Boron - Mineralogy, petrology and geochemistry. Rev Mineral 33: 559-643.

Slack JF, Herriman N, Barnes RG and Plimer IR. 1984. Stratiform tourmalines in metamorphic terranes and their geologic significance. Geology 12: 713-716.

Taylor BE and Slack JF. 1984. Tourmalines from the Appalachian-Caledonian massive sulfide deposits: textural, chemical, an isotopic relationships. Econ Geol 79: 1703-1726.

TAYLOR JR HP. 1967. Oxygen isotope studies of hydrothermal mineral deposits. In: BARNES HL (Ed.), Geochemistry of hydrothermal ore deposits. Holt, Rinehart and Winston, 670p.

Willner AP. 1992. Tourmalines from the stratiform peraluminous metamorphic suite of the Central Namaqua Mobile Belt (South Africa). Mineral Deposita 27: 304-313.

YAVUZ F. 1997. TOURMAL: Software package for tourmaline, tourmaline-rich rocks and related ore deposits. Computers and Geosciences 23: 947-959. 